\title{
1 CRISPR/Cas9-mediated genome editing of Schistosoma mansoni acetylcholinesterase
}

2
Hong You ${ }^{1 *}$, Johannes U. Mayer ${ }^{2}$, Rebecca L. Johnston ${ }^{3}$, Haran Sivakumaran ${ }^{3}$, Shiwanthi Ranasinghe $^{1}$, Vanessa Rivera ${ }^{1,4}$, Olga Kondrashova ${ }^{3}$, Lambros T. Koufariotis ${ }^{3}$, Xiaofeng Du$^{1}$, Patrick Driguez ${ }^{5}$, Juliet D. French ${ }^{3}$, Nicola Waddell ${ }^{3}$, Mary G. Duke ${ }^{1}$, Wannaporn Ittiprasert ${ }^{6}$, Victoria H. Mann ${ }^{6}$, Paul J. Brindley ${ }^{6}$, Malcolm K. Jones ${ }^{1,7}$, Donald P. McManus ${ }^{1 *}$

1. Immunology Department, QIMR Berghofer Medical Research Institute, Brisbane, 4006, Australia

2. Malaghan Institute of Medical Research, Wellington, 6012, New Zealand

3. Genetics \& Computational Biology Department, QIMR Berghofer Medical Research Institute, Brisbane, 4006, Australia

4. School of Medicine, Deakin University, Geelong, 3216, Australia

5. King Abdullah University of Science and Technology, Thuwal 23955-6900, Kingdom of Saudi Arabia

6. Department of Microbiology, Immunology \& Tropical Medicine, \& Research Center for Neglected Diseases of Poverty, School of Medicine \& Health Sciences, George Washington University, Washington, D.C. 20037, USA

7. School of Veterinary Science, The University of Queensland, Gatton, 4343, Australia

Corresponding authors: Hong You

Hong.You@qimrberghofer.edu.au

Donald P. McManus Don.McManus@ qimrberghofer.edu.au

Key words: Schistosoma mansoni, Schistosome eggs, CRISPR/Cas9, Genome editing, Acetylcholinesterase, Synthetic guide RNA, Single-stranded oligodeoxynucleotides, Granuloma, Cytokines

Short title: CRISPR/Cas9-mediated genome editing system in Schistosoma mansoni 


\section{Abstract}

CRISPR/Cas9-mediated genome editing shows cogent potential for the genetic modification of helminth parasites. Here we report successful gene knock-in (KI) into the genome of the egg of Schistosoma mansoni by combining CRISPR/Cas9 with single-stranded oligodeoxynucleotides (ssODNs). We edited the acetylcholinesterase (AChE) gene of $S$. mansoni targeting two guide RNAs (gRNAs), X5 and X7, located on exon 5 and exon 7 of Smp_154600, respectively. A CRISPR/Cas9-vector encoding gRNA X5 or X7 was introduced by electroporation into eggs recovered from livers of experimentally infected mice. Simultaneously, eggs were transfected with a ssODN donor encoding a stop codon in all six frames, flanked by 50 nt-long 5'- and 3'homology arms matching the predicted Cas9-catalyzed double stranded break at X5 or X7. Next generation sequencing analysis of reads of amplicon libraries spanning targeted regions revealed that the major modifications induced by CRISPR/Cas9 in the eggs were generated by homology directed repair (HDR). Furthermore, soluble egg antigen from AChE-edited eggs exhibited markedly reduced AChE activity, indicative that programmed Cas9 cleavage mutated the AChE gene. Following injection of AChE-edited schistosome eggs into the tail veins of mice, a significant decrease in circumoval granuloma size was observed in the lungs of the mice. Notably, there was an enhanced Th2 response involving IL-4, -5, -10, and-13 induced by lung cells and splenocytes in mice injected with X5-KI eggs in comparison to control mice injected with unmutated eggs. A Th2-predominant response, with increased levels of IL-4, -13 and GATA3, also was induced by X5 KI eggs in small intestine-draining mesenteric lymph node cells when the gene-edited eggs were introduced into the subserosa of the ileum of the mice. These findings confirmed the potential and the utility of CRISPR/Cas9-mediated genome editing for functional genomics in schistosomes.

\section{Author Summary}

Schistosomiasis is the most devastating of the parasitic helminth diseases. Currently, no vaccines are available for human use and praziquantel is the only available treatment raising considerable concern that drug resistance will develop. A major challenge faced by the schistosomiasis research community is the lack of suitable tools to effectively characterise schistosome gene products as potential new drug and/or vaccine targets. We introduced CRISPR/Cas9 mediated editing into $S$. mansoni eggs targeting the gene encoding acetylcholinesterase (AChE), a recognized anthelminthic drug target. We found that the major modifications induced by CRISPR/Cas9 in the eggs were generated by homology directed repair (HDR). This platform provides a unique opportunity to generate precise loss-of-function insertions into the schistosome genome. We pre-screened the activity of two guide RNAs of the AChE gene and

67 compared/validated the mutation efficacy using next-generation sequencing analysis at the genomic level and phenotypic modifications at the protein level. That resulted in reduced AChE activity observed in AChE-edited eggs, and decreased lung circumoval granuloma size in mice

70 injected with those edited eggs. The CRISPR/Cas9-genome editing system we established in this study provides a pivotal platform for gene functional studies to identify and test new anti- 
schistosome intervention targets, which can be extended to the other human schistosome species and other important parasitic helminths.

\section{Introduction}

Schistosomiasis remains one of the most prevalent, chronic and insidious of the tropical parasitic diseases. Human infection begins when larval schistosome cercariae penetrate the skin. Once in the bloodstream, the cercariae transform into schistosomula which migrate to venules associated with specific organs (e.g. liver, intestine, bladder) where they mature as adult male and female worms and rapidly start to reproduce. Their eggs release molecules that damage host tissues through induction of extreme granulocytic responses and tissue scarring. No effective schistosomiasis vaccine is available and treatment is entirely dependent on a single drug, praziquantel (PZQ) [1]. Despite the wide-spread use of PZQ, with over 40 years of mass drug administration programs, the number of people infected, particularly in Africa, has not decreased substantially and, in fact, may exceed published figures [2]. Furthermore, this MDA strategy is unsustainable long term and the spectre of the likely generation of PZQ-resistant parasites is a constant threat. Complete genomic sequences are available for Schistosoma japonicum [3], $S$. mansoni [4] and S. haematobium [5]. The complex, multi-generational life cycles of schistosomes and their recalcitrance for genetic manipulation has resulted in major difficulties for gene editing [6]. Notably, the paucity of molecular tools to manipulate schistosome gene expression has markedly limited our ability to define key metabolic pathways, thereby hampering discovery of new anti-schistosome drugs or vaccines.

CRISPR/Cas9 is an advanced genome editing tool and RNA-guided system whereby a 20-base guide RNA (gRNA) directs the Cas9 nuclease from Streptococcus pyogenes to cleave a target gene, generally providing high specificity and minimal off-target site effects. The endonuclease makes a double-strand break (DSB) at the target site [7]. Endogenous DSBs in eukaryotes are repaired by at least two repair mechanisms [8]: non-homologous end joining (NHEJ), which results in nucleotide insertions and deletions [9]; and homology directed repair (HDR) which utilizes a repair template. In terms of heterogeneity of indels introduced at Cas9-induced DSBs, allelic editing frequencies are variable [10]. The prokaryotic CRISPR/Cas9 system was first adapted for Caenorhabditis elegans in 2013 [11] and used to generate transgenic organisms and introduce site-specific, heritable mutations [12]. It was further extended for genome editing in diverse organisms, including human protozoan parasites Toxoplasma gondii, Plasmodium falciparum, Trypanosoma cruzi and Leishmania species [13]. Deployment of the CRISPR/Cas9 system for genome editing [14] extends the range of experimental approaches to interrogate the

108 host-parasite relationship and test novel vaccines or drugs. Post-transcriptional gene silencing 109 (RNA interference), which has been developed over the last 15 years for loss-of-function 110 research in schistosomes, is transient and limited in maintaining inheritance of the targeted gene. 111 Ittiprasert et al pioneered the CRISPR/Cas9 editing system in S. mansoni eggs, targeting a single 
112 gRNA of omega-1 ( $\omega 1)$, which plays a crucial role in Th2 polarization and granuloma formation 113 [15]. Sankaranarayanan et al compared CRISPR/Cas9 mediated deletions in different life cycle 114 stages of S. mansoni including adult worms, sporocysts and eggs, targeting the gene SULT-OR 115 which is involved in oxamniquine resistance [16] (preprint data). Arunsan et al. also undertook a 116 similar approach to modify the granulin $(O v$-GRN-1) gene in the liver fluke Opisthorchis 117 viverrini which plays a key role in virulence morbidity during opisthorchiasis [17]. These pivotal 118 studies demonstrated that programmed genome editing is feasible in flatworm parasites.

Effective site-specific gene modification and phenotyping will drive innovation and a better understanding of schistosome pathogenesis, biology and evolution [6]. To expand functional genomic investigations of schistosomes and, in particular, to advance our understanding of the function of acetylcholinesterase (AChE) in these blood flukes, we undertook CRISPR/Cas9mediated knock-out (KO) and knock-in (KI) in adult S. mansoni and eggs targeting this key enzyme. AChE is the target of a number of currently approved and marketed anthelmintics [1820]. Furthermore, AChE plays an important role in the adult schistosome neuromusculature cholinergic system [21, 22], and is intimately involved in muscle function [23, 24] and other essential activities such as feeding, sexual maturation and mating in mature worms [24]. We previously demonstrated that $\mathrm{AChE}$ is present on the external tegumental membrane and in the musculature of adult schistosomes [21, 25]. AChE also occurs within the eggs and in the host cellular components of the advanced granulomas around the parasite eggs entrapped in various organs [26] indicating an involvement in granuloma formation as a result of its role in inhibiting the host IL-4 response [26].

Here, we used programmed genome editing to modify the AChE-encoding locus in the genome of the eggs and adult developmental stages of S. mansoni using CRISPR/Cas9-based KO and KI approaches. We determined the efficiency of CRISPR/Cas9-mediated midifications by next generation sequencing analysis (NGS) on genomic DNA extracted from mutated parasites. We compared KO/KI efficacy by targeting two gRNAs of AChE distanced with $14.3 \mathrm{~kb}$ genomic sequence individually and in combination. In addition, we assessed in vivo immune responses induced by AChE-KI eggs in lung cells, splenocytes and small intestine-draining mesenteric lymph node cells following injection of AChE KI eggs into the tail vein of mice or into the small intestinal subserosa of mice.

\section{Results}

148 Two guide RNAs were designed to target residues $722-741$ in exon 5 (X5) and $1738-1757$ in 149 exon 7 (X7), respectively, of the acetylcholinesterase gene (AChE, Smp_154600, Uniprot 150 Q71SU7_SCHMA), which is located on S. mansoni chromosome 1 (Fig 1A). The nucleotide 151 residues complementary to guide RNA-X5 and -X7 are adjacent to the protospacer adjacent 
152 motifs (PAM), TGG and AGG, respectively, with the predicted Cas 9 cleavage site located three

153 residues upstream of the PAM. These PAMs (TGG or AGG) and the nucleotide sequences

154 complementary to these two gRNAs are highly specific and are absent from two paralogues of

155 Smp_154600, i.e., Smp_136690 and Smp_125350. These two paralogues share 34-38\%

156 nucleotide identity and 25-26\% amino acid identity to Smp_154600. The Smp_136690 and

157 Smp_125350 genes exhibit a distinct exon/intron structures (S1 Fig) (although this may reflect

158 imprecise annotation of the draft genome sequence). S2 Fig presents the amino acid sequence

159 alignment and predicted functional motifs of Smp_154600, Smp_136690 and Smp_125350.

160

161

Site-specific integration of exogenous DNA confirms CRISPR/Cas9 activity in schistosomes

162

163

We investigated the activity and efficiency of programmed gene knockout (KO) of the AChE gene (Smp_154600) of S. mansoni. The two AChE locus specific gRNAs (X5 and X7 described above) were cloned into the GeneArt CRISPR Vector, which encodes Cas9 of Streptococcus pyogenes driven by the human cytomegalovirus (CMV) promoter and gRNA (X5 or X7) driven by the human U6 promoter. Freshly perfused $S$. mansoni adult worms and eggs isolated from the livers of infected mice were transfected with the plasmid vector constructs by square wave electroporation.

Homology directed repair (HDR) of CRISPR/Cas9-induced double stranded breaks (DSBs) at a gene locus in the presence of donor DNA template was reported in the human threadworm Strongyloides stercoralis [27]. Accordingly, as a template for HDR of chromosomal DSBs, a single stranded oligodeoxynucleotide (ssODN) of $124 \mathrm{nt}$ in length targeting X5 (X5ssODN, Fig 1B) was delivered with the X5-CRISPR vector (X5 knock-in, $\mathrm{X} 5$-KI) and ssODN targeting $\mathrm{X} 7$ (X7ssODN, Fig 1C) was delivered with X7-CRISPR vector (X7-KI); both X5ssODN and $\mathrm{X} 7 \mathrm{ssODN}$ were delivered with X5-CRISPR vector and X7-CRISPR vector (X5+X7-KI) into eggs and adult worms.

Both $\mathrm{X} 5 \mathrm{ssODN}$ and $\mathrm{X} 7 \mathrm{ssODN}$ include a short transgene encoding six stop codons flanked by 5 'and 3'-homology arms, with each arm $50 \mathrm{nt}$ in length complementary to the genome sequence of exon 5 and 7, respectively, on both the $5^{\prime}$ and 3 ' sides of the programmed Cas 9 cleavage site (Fig 1B, 1C). Given that the donor ssODN includes a short transgene in order to facilitate genotyping, PCR was performed using template genomic DNA extracted from the CRISPR/Cas9-treated eggs and adults to detect the extent of KI. Targeting AChE gRNA X5, a reverse primer termed $\mathrm{R}$, specific for the stop codon region of the donor ssODNs, was paired with two discrete forward primers (termed CF and F2, Fig 1D); R4 was designed as a reverse primer to pair with $\mathrm{CF}$ and F2 as positive (wild type) control (Fig 1D). Targeting gRNA X7, a forward primer termed F, specific for the stop codon region of the donor ssODN transgene, was paired with two discrete reverse primers (termed R1 and R6, Fig 1D); F1 was designed as forward primer to pair with R1 191 primers. 
At AChE X5 amplicons of the expected sizes for primer pairs $\mathrm{CF}+\mathrm{R}(682 \mathrm{bp}$ ) and $\mathrm{F} 2+\mathrm{R}$ (307 bp) were observed in eggs (Fig 1E) and adults (S3A Fig) in genome-edited knock in (KI) groups $\mathrm{X} 5 \mathrm{KI}$ and $\mathrm{X} 5+\mathrm{X} 7-\mathrm{KI}$, but not in group X7-KI, indicating successful insertion of the ssODN transgene and resolution of the DSB. PCR results from the control groups, namely parasites bp or 307 bp. AChE X7 amplicons of 392 bp (primers F+R1) were observed in groups X7-KI and X5+X7-KI in both eggs (Fig 1E) and adults (S3 Fig), but not in group X5-KI and the CON groups (Fig 1E), indicating programmed insertion of the ssODN transgene. The PCR profiles obtained for groups of parasites treated with ssODN only (including X5ssODN and X7ssODN) were the same as the control groups. Importantly, Sanger sequencing analysis of the KI amplicons confirmed the insertion of the transgene into the AChE locus at X5 and X7 at the predicted cleavage sites. The viability of eggs isolated from livers of mice infected with S. mansoni cercariae and postelectroporation revealed hatching rates of $70-80 \%$ (S4 Fig). The hatching rate was slighted decreased, but not significantly $(p=0.16)$, in eggs subjected to electroporation compared with those that were not (S4 Fig), indicating that electroporation did not markedly affect egg viability. Microscopy confirmed adult worms of S. mansoni, cultured for two days post-electroporation, were alive.

Programmed modifications at exons 5 and 7 of the AChE locus within the genome of the eggs of $S$. mansoni

To characterize and quantify the modifications at the nucleotide level resulting from CRISPR/Cas9-induced AChE-KO/I into the schistosome egg, we used an amplicon NGS approach, with specific forward primers (F2 for X5 and F1 for X7, Fig. 1D) and reverse primers (R4 for X5 and R6 for X7, Fig. 1D) designed to target the AChE locus in regions flanking the predicted programmed modifications. Barcoded amplicon libraries were constructed from pooled genomic DNA of independent groups of eggs with respect to the several guide RNAs and donor ssODN combinations. The sequencing was performed on the Illumina MiSeq platform. CRISPResso2 [28] was used to analyse deep-coverage sequence reads. On average, 312,000 reads per sample aligned to the respective reference amplicon sequence of the Smp_154600 locus (S2 Table).

\section{Modifications observed at AChE exon 5}

We used the CRISPResso2 software to analyze the sequenced amplicon reads obtained using the primers F2 and R4 targeting exon 5 of AChE. After filtering the reads based on the F2+R4 primers, $0.21 \%$ of reads from eggs subjected to X5-KI treatment exhibited sequence variations ascribable to chromosomal DSB repair by NHEJ or HDR. By running CRISPResso2 with an 
232 increasing window size parameter (value of 1,20, 100, and 0) which defines the size (in bp) of 233 the quantification window extending from the DSB, we found that the amount of NHEJ 234 modifications, particularly NHEJ substitutions, also stably increased across both the negative 235 control and experimental samples (see S5 Fig). Therefore, we kept the quantification window 236 size around each gRNA to the default value of 1 to limit the amount of PCR and/or sequencing 237 errors from being inappropriately quantified as modified reads and we found that the majority 238 (>95\%) of modified reads with NHEJ were due to substitutions, and not insertions or deletions 239 (see S2 Table). Of the reads that were modified in the samples treated with X5-KI, 0.14\% were 240 confirmed by fasta36 to be HDR modified reads (Fig 1F). We also analysed samples treated with 241 X5+X7-KI and found $0.04 \%$ of reads were confirmed HDR events, which is lower than the 242 percentage of HDR reads in samples treated only with X5-KI (Fig 1F). Additionally, we detected 243 a small number of HDR events in the negative control samples which may have been due to 244 sample cross-contamination possibly occurring during barcoding in the amplicon library 245 preparation. The level of background noise for HDR detection was set to $0.014 \%$, since the 246 negative control samples contained HDR events below this value. By subtracting the level of 247 background noise for HDR detection at exon 5 from the rate of confirmed HDR in X5-KI $248(0.14 \%)$ and $\mathrm{X} 5+\mathrm{X} 7-\mathrm{KI}(0.04 \%)$, we estimated that $0.12 \%$ and $0.03 \%$ of reads in the X5 experimental samples carried CRISPR/Cas9-induced HDR events, respectively.

\section{Modifications at AChE exon 7}

We used CRISPResso2 to analyse the raw reads from samples that were amplified using the F1+R6 primers targeting AChE exon 7. After filtering reads based on primers F1+R6, $0.26 \%$ of reads from the sample treated with X7-KI were found modified by NHEJ or HDR: $0.13 \%$ were confirmed as HDR events by fasta36 analysis, while the other $0.13 \%$ were NHEJ modifications, majority of which were substitutions and not insertions or deletions (see S2 Table). Similar to the findings with exon 5, the samples treated with X5+X7-KI contained fewer confirmed HDR events $(0.04 \%)$ than samples treated with the single guide and donor combination (X7-KI). For exon 7, the level of background noise for HDR detection was set to $0.0105 \%$, since the negative control (X7 and X7ssODN groups) samples contained HDR events below this value. By subtracting the level of background noise for HDR detection at exon 7 from the rate of confirmed HDR in the experimental samples, we estimated that $0.1195 \%$ and $0.0295 \%$ of reads represented CRISPR/Cas9-induced HDR events in the X7 treatment samples, respectively.

\section{Decreased AChE activity in CRISPR/Cas9-mediated KI eggs and adult worms}

The AChE activities of soluble egg antigen (SEA) extracted from eggs, and soluble worm antigen preparation (SWAP) obtained from adult worms of S. mansoni, were determined following CRISPR/Cas9-mediated AChE-KI. Significant decreases in AChE activity were observed in SEA (Fig 2A) extracted from eggs treated with X5-KI (10.7\%, $p=0.001$ ), X7-KI $(8.3 \%, p=.006)$ and X5+X7-KI $(13.4 \%, p=0.0002)$ compared with SEA from control eggs electroporated with CON. Remarkable reductions in AChE activity were also detected in SWAP 
(S3B Fig) extracted from adults treated with X5-KI (21\%, $p=0.023)$, X7-KI (11.8\%, ns) and $\mathrm{X} 5+\mathrm{X} 7-\mathrm{KI}(23.8 \%, p=0.012)$ compared with control adults electroporated with CON. The control groups, including SEA (or SWAP) extracted from wild type parasites and parasites electroporated with media, CON or ssODN only, exhibited a similar level of AChE activity, and no significant differences were observed amongst them.

\section{Inhibition of migration of LX-2 cells in vitro by SEA isolated from AChE-KI eggs} It is recognized that hepatic stellate cells (HSC) resident within liver granulomas are critical players involved in hepatic schistosomiasis being one of the major cell types responsible for the egg-induced immunopathology and resulting fibrosis $[29,30]$. To determine further phenotypic changes induced in eggs by AChE-KI we examined the effect of treating LX-2 cells with SEA isolated from AChE-KI eggs, by scratch wound assay. LX-2 is a developed immortalised human HSC cell line that exhibits the key features of primary HSC [31]. Real time monitored cell growth assays using the IncuCyte system were undertaken to test the effects of SEA isolated from AChE-KI and control eggs on the proliferation of LX-2 cells. Cell growth images on days 0, 1 and 2 post-incubation with SEA extracted from mutated/unmutated eggs are shown in S6 Fig. The scratch wound assay revealed that, after 3 days culture with SEA $(30 \mu \mathrm{g} / \mathrm{ml})$, the wound width of LX-2 cells was significantly larger in cells subjected to SEA extracted from eggs treated with X5-KI, X7-KI or X5-KI+X7-KI compared with SEA from eggs electroporated with EV (or medium or WT eggs) (Fig 2B). Our analysis showed that the rate of scratch wound closure in SEA-treated cells decreased by $22 \%$ in X5-KI SEA $(p=0.002), 18 \%$ in X7-KI SEA $(p=0.01)$ and $14 \%$ in X5+X7-KI SEA $(p=0.03)$ compared with the CON SEA (Fig 2B). These data show that SEA derived from CRISPR/Cas9-modified KI eggs targeting either AChE X5 or X7 significantly inhibited HSC migration.

Decreased granulomatous inflammation and collagen expression in lungs of mice injected with CRISPR/Cas9-mediated AChE-KI eggs AChE-KI (X5-KI, X7-KI and X5+X7-KI) eggs were injected into the lateral vein of the tails of mice to determine whether AChE-KI eggs affected the development of granulomas associated with pulmonary schistosomiasis in vivo. In this model, eggs are transported to the lungs via the bloodstream resulting in subsequent granuloma formation [32]. Two weeks post-injection, mice were euthanized and the lungs of each animal were removed for histological analysis. Representative digital microscopic images of lung tissues acquired through the Aperio ImageScope software are shown in Fig 2C. Notably, decreased granuloma area was observed in the AChE-KI (X5-KI, X7-KI and X5+X7-KI) groups (Fig 2F). The relative areas of granulomas surrounding individual eggs were quantified and showed 4.3-fold $(p=0.006), 8.2$-fold $(p<0.0001)$ and 10 -fold $(p<0.0001)$ reductions in the $\mathrm{X} 5$-KI group, $\mathrm{X} 7-\mathrm{KI}$ and $\mathrm{X} 5+\mathrm{X} 7-\mathrm{KI}$ group, respectively, compared with the size of granulomas formed around eggs electroporated with CON (Fig 2C). These results documented a marked deficiency in the induction of pulmonary granulomas by the injected AChE-KI eggs compared with CON eggs of S. mansoni. However, 
312 we noted a significantly increased granuloma area detected in the WT group compared with

313 those in the Medium/CON groups (Fig 2C, 2E), suggesting that electroporation may have 314 contributed to the reduced egg fitness in the infected mice. During granuloma development in 315 liver, activated hepatic stellate cells deposit a ring of collagen to encapsulate what then becomes 316 the 'core' of the granuloma. We thus used portions of the mouse lung to assess the transcription 317 levels of collagen (collagen type 1, alpha 1-COL1 $\alpha 1$ ) in the AChE-KI groups by qPCR (Fig 2D). 318 There were significant decreases in collagen mRNA expression in the mice injected with X5-KI 319 eggs $(75 \%, p=0.036)$, X7-KI eggs $(46 \%, p<0.0001)$ and $\mathrm{X} 5+\mathrm{X} 7-\mathrm{KI}$ eggs $(59 \%, p=0.005)$ 320 compared with control eggs treated with CON only (Fig 2D). No statistical difference in 321 collagen mRNA expression was observed in the lungs of mice injected with WT eggs or injected eggs treated with $\mathrm{CON}(p>0.05)$.

\section{Serum IgE levels decreased in mice injected intravenously (i.v.) with X5-KI eggs}

As a marker of Th2 response polarization in schistosome infection [33], mouse IgE levels were measured in serum samples collected from mice two weeks post-iv-injection with eggs treated with X5-KI, X7-KI or X5+X7-KI. A significant decrease in the level of IgE $(p=0.04)$ was observed in mice injected with X5-KI eggs, but not X7-KI or X5+X7-KI eggs, compared with animals injected with CON treated eggs (Fig 2E).

\section{Cytokine responses generated in lung cell isolates and splenocytes of mice injected i.v. with AChE-KI eggs}

To further evaluate the immune response generated in mice by AChE-KI eggs, cytokines secreted from lung cells and splenocytes were quantified using LEGEND plex mouse Th1/Th2 panel kits. Both lung cells and splenocytes isolated from individual mice at two weeks post egginjection were incubated with SEA extracted from normal S. mansoni liver eggs for 72 hours. Culture supernatants were then collected for cytokine population analysis. We found that the levels of IL-2, 4, 5, 10, 13 in lung cell (Fig 3A) and IL-4, 5, 6, 10, 13 in splenocytes (Fig 3B) were significantly increased in mice injected with X5-KI eggs in comparison to control mice injected with CON-eggs. Significantly enhanced levels of IL-6 and TNF $\alpha$ in splenocytes and $\mathrm{TNF} \alpha$ in lung cells were observed in mice injected with X7-KI eggs (Fig 3A). However, significant change in cytokine response was not evident in the $\mathrm{X} 5+\mathrm{X} 7-\mathrm{KI}$ egg injected group.

\section{IL-4, IFN $\gamma$, Ctla4 and RetnlA mRNA expression in lungs of mice injected i.v. with AChE- KI eggs}

To determine whether AChE-KI eggs would change the alternative activation of macrophages, an important hallmark of innate type 2 immunity [34], qPCR was performed on lungs from i.v. injected animals; the expression of IL-4 and IFN $\gamma$, Ctla4 (cytotoxic T-lymphocyte-associated protein 4) and RetnlA (resistin-like molecule alpha) were also measured. We found increased levels of IL-4 in mice injected with X5-KI (S7A Fig) and enhanced Ctla4 responses were detected in mice injected with X7-KI eggs compared with mice injected with CON-eggs (S7 
Fig). However, a significant decline in the level of IL-4 was observed in mice injected with CON eggs compared with those injected with WT-eggs (S7A Fig). No significant difference was observed in the expression of IFN $\gamma$ or RetnlA in lungs of mice injected with AChE-KI eggs compared with those receiving CON eggs (S7B and S7C Fig).

Immune responses in mouse mesenteric lymph node (MLN) cells induced by intestinal injection of AChE-KI eggs

To address effects on intestinal immune responses, which have been linked to egg migration and pathogenesis [35], AChE-KI (or unmutated) eggs were injected into the subserosal layer of the ileum of mice, as previously described [36]. The ileum draining mesenteric lymph node (MLN) cells were isolated from mice on day 5 post injection and divided into two groups to undertake flow cytometric analysis and to quantify secreted cytokines (IFN $\gamma$, IL-4 and IL-13) in culture supernatants following 72 hours culture

Flow cytometric analysis did not reveal significant difference in the number of CD4+ T cells isolated from mice injected with AChE-KI eggs compared with those injected with eggs electroporated with CON (Fig 3C). However, the number of activated CD44hi CD4+ T cells, GATA3+ and IL-4+ T cells were significantly increased in mice injected with eggs treated with X5-KI (Fig 3); increased cell numbers of CD4+GATA3 and CD4+IL-4 cells were observed in mice injected with X7-KI eggs compared with mice injected with CON eggs. S8 Fig presents the gating strategy for flow cytometric quantification of IFN $\gamma$, GATA3, IL-4 and IL-13 levels produced by MLN cells.

The levels of secreted cytokines (IL-4, IL-13 and IFN- $\gamma$ ) were also determined in supernatants of MLN cells after in vitro culture for 72 hours. The levels of IL-4 and IL-13 secreted by MLN cells isolated from mice injected with eggs treated with X5-KI were significantly enhanced compared with those from control mice injected with $S$. mansoni CON eggs (Fig 3C). In contrast, no change was observed in the level of IFN $\gamma$ generated by MLN cells isolated from mice injected with treated or untreated eggs (Fig 3C).

\section{Discussion}

We demonstrated CRISPR/Cas9-mediated site-specific AChE modification in S. mansoni eggs. Given gRNAs with different target sites might guide different endonuclease activities [37, 38], we first pre-screened the activity of two gRNAs (X5 and X7) combined with/without appropriate ssODNs within the AChE loci. Importantly, we found the major modification induced by the CRISPR/Cas9-mediated editing in eggs was HDR. The effect of this modification was further reflected by the in vivo immune responses induced by AChE-KI eggs in different tissues of mice, 
In our NGS analysis of amplicons from AChE-KI eggs we found, using CRISPResso2 software and a $1 \mathrm{bp}$ window size, that the majority (>95\%) of modified reads with NHEJ were due to substitutions, and not insertions or deletions. Furthermore, we determined that the frequency of NHEJ substitutions increased equally across all analysed samples, including control groups, when the window size was changed from 1 bp to the entire amplicon length. This suggests the NHEJ substitutions detected by CRISPResso2 are likely false positives introduced through PCR or sequencing errors although further study is required to confirm these observations. Deletions and insertions were rare in AChE-edited eggs, similar to the report by Sankaranarayanan et al. [16], where CRISPR/Cas9 was used to edit the SULT-OR in S. mansoni eggs. A possible explanation for these results might be low expression levels of some key NHEJ repair enzymes required for CRISPR/Cas9 editing in schistosome eggs [16], including gene Smp_211060 identified in $S$. mansoni as a homolog of the KU70/KU80 genes which are essential for the NHEJ pathway [39-41]. The limited NHEJ indels detected in AChE-edited eggs suggested the possibility that most DSBs in AChE loci can be repaired by re-ligating directly without insertion or deletions. However, when ssODN was provided, the DSBs generated by CRISPR/Cas9 editing were repairable by HDR, which is a different outcome to that observed with vertebrate cells where the HDR of the DSBs is extremely low compared to NHEJ, at least where both pathways are equally available [42]. In some single celled parasites including Plasmodium [4346], Trichomonas vaginalis [47] and Cryptosporidium parvum [48], HDR is the only mechanism of DSB repair due to the lack of the NHEJ pathway; this leads to more accurate repair with less unintended or off-target effects. However, in Toxoplasma gondii, frequent NHEJ of DSBs occurs, resulting in random modifications $[49,50]$. Precise custom modifications via HDR in $T$. gondii are generated by disrupting the NHEJ pathway through deleting KU80, the key gene for NHEJ [39-41], leading to more specific gene editing. Our study shows that the CRISPR/Cas9 mediated editing system in schistosome eggs (delivered by electroporation) was able to induce HDR more prominently; this may provide a mechanism to generate loss-of-function insertions into site-specific nucleases in the schistosome genome and afford a new approach for studying gene editing in blood flukes.

HDR efficiency can be utilized to increase gene ablation [51] reflected by a marked reduction in protein activity. The reduced AChE activity detected in AChE-KI eggs of S. mansoni, together with the modified immune response in mice induced by AChE-KI eggs, suggest that the amount of modification ( 0.12 by HDR mediated repair) observed in the genomic NGS analysis, albeit small, nevertheless led to a pronounced phenotypic change at the protein level in this multicellular parasite. The other recent CRISPR/Cas9 studies on parasitic flatworms [52], including targeting $\omega 1$ in S. mansoni [15] and Ov-grn-1 in O. viverrini [17], reported similar findings. Schistosomes are complex, multi-cellular, multi-organ invertebrates that have evolved a range of adaptations to survive in diverse environments, and these features present considerable challenges for successful genomic editing. Incomplete modification of the AChE gene may stimulate $S$. mansoni to alter cholinergic signalling transduction and further regulate downstream 
signalling pathways (such as the PI3K and ERK pathways), thereby amplifying the effect of the knockdown. In addition, as reported in CRISPR/Cas9 editing in S. mansoni [16], Strongyloides [53] and C. elegans [54], large deletions might be missed by amplicon sequencing. Undetected modification(s) during AChE editing may have led to the pronounced phenotypic changes observed at the protein level in these helminths but this needs to be further explored.

The low efficacy of CRISPR/Cas9 mediated gene editing of AChE achieved in S. mansoni eggs may be due to particular characteristics of this multicellular parasite now considered.

1. The schistosome egg has a protective eggshell with a hardened structure comprising three layers [55] that may make it difficult to penetrate [56]. Nevertheless, targeting the egg may provide optimal access to schistosome germ line cells in order to introduce insertional mutagenesis in chromosomes of the blood fluke [57]; this is because the egg has a relatively simple structure and a high stem-like cell content [58] compared with other life cycle stages,

2. Using a pool of several thousands of liver eggs for CRISPR/Cas9-mediated editing may result in low knockdown efficacy. Liver eggs represent a mixture of eggs of variable age and different stages of development and in S. mansoni higher transgene efficiency at the genomic level occurs in immature compared with mature eggs [57]. The use of freshly laid eggs by adult schistosomes cultured in vitro may improve on the knockdown effect but the logistics involved in collecting sufficient numbers of these immature eggs for downstream sequencing analysis and phenotype studies present a major challenge.

3. Variation in the pattern of CRISPR/Cas9 induced modifications may depend on the function or distribution of the targeted gene. The AChE protein is located throughout different components of schistosome eggs [26] and is highly expressed in mature eggs (S9 Fig) in which the miracidia have developed within the eggshell into multi-cellular, mobile, ciliated larvae composed of organs, tissues, muscles and nerves. CRISPR/Cas9-mediated AChE editing in the nuclei of cells within the thin cellular epithelia of individual mature eggs may have been more frequent than in cells deeper within the egg including the extra-embryonic inner envelope. When AChE-KI eggs were injected into mice, the edited AChE distributed in the egg epithelia would be readily released from the eggs into the surrounding tissues thereby modulating host immune responses.

In this study, our NGS analysis focused on AChE-edited eggs. However, as reported by Sankaranarayanan et al [16], adult S. mansoni might be a better stage to achieve higher efficiency of CRISPR/Cas9 mediated modifications compared with eggs (and sporocysts). Accordingly, an improved programmed genome editing may occur in AChE-edited adults, which we now plan to investigate.

Schistosome eggs play critical roles in host pathogenesis, immune modulation, and the transmission of schistosomiasis. Eggs trapped in the intestinal wall or liver can induce a strong Th2 immune response which is associated with the chronic pathology characteristic of 
schistosomiasis [59]. A novel mouse infection model, involving intravenous injection of eggs via the tail vein, revealed enhanced Th2 responses in lung cells and splenocytes isolated from mice injected with X5-KI eggs. The role of S. mansoni X5-KI eggs in suppressing the Th2 immune response was further confirmed when intestinal injection of these mutated eggs was performed, a superior method for assessing the generation of specific anti-egg immune responses. MLNs isolated from the intestine, where eggs are locally injected, provide a more sensitive readout of generated immune responses and the approach more closely resembles the natural site of egg transition and granuloma formation [35]. The MLN analysis indicated that knockdown of AChE targeting gRNA X5 in eggs could trigger a superior host immune response than that targeting gRNA X7, although NGS analysis of X5-KI and X7-KI eggs showed similar levels of HDR modifications $(\sim 0.12 \%)$. The upstream location of gRNA X5 in the genome compared with X7 allowed insertion of stop codons delivered by X5ssODN into the DSB of X5 generated by CRISPR/Cas9 editing, thereby affecting the expression of downstream DNA sequences of AChE. This further suggests that motifs or domains of AChE that are involved in regulating the Th2 immune response might be located between the X5 and X7 sites in the AChE gene.

The important role of cholinergic signalling in a helminth parasite influencing the host immune system was recently reported in a study where Trypanosoma musculi was engineered to heterogeneously express a secreted AChE from Nippostrongylus brasiliensis; the secreted protein was shown to alter the cytokine environment by enhancing production of IFN $\gamma$ and TNF $\alpha$, with a concomitant reduction in IL-4, IL-5 and IL-13 levels [60]. We also previously showed a predominantly Th1-type protective immune response characterized by increased production of IFN $\gamma$ in mice vaccinated with recombinant AChE [26] following schistosome challenge, indicating egg-secreted AChE might be critical in inhibiting the host IL-4 response. These prior studies support the findings reported here where X5-KI eggs elicited an increased Th2 response characterized by elevated levels of IL-4, IL-5, IL-10 and IL-13 secreted by splenocytes. These cytokines are predominantly produced by Th2 cells in various models of type 2 immunity [61, 62] and they inhibit Th1 responses [63]. This may account for the reason why we did not observe any changes in the levels of IL-2 and IFN $\gamma$ which are characteristically produced by Th1 cells [64]. To confirm in vivo alveolar immune responses induced by the AChE-KI eggs in mice, lung homogenates were used in qPCR assays to determine the mRNA expression of IL-4, INF $\gamma$, Ctla4 and RetnlA. The level of IL-4 was increased only in mice injected with X5-KI eggs complementing the significantly increased level of IL-4 observed in the lung cells and splenocytes. The expression of Ctla4, which inhibits the Th2 response [65], was increased in mice injected with $\mathrm{AChE}-\mathrm{X} 7 \mathrm{KI}$ eggs, and this was reflected by the increased level of TNF (typifying a Th1 response) observed in splenocytes isolated from mice injected with AChE-X7 KI eggs. RetnlA is known to be involved in the down modulation of Th2 responses [66] and is expressed by alternatively activated macrophages [66]. However, there was no significant difference observed in the RetnlA expression in all groups, indicating that AChE- 
511 KI in eggs may be associated with Ctla4-mediated Th2 suppression, or that the effects were too 512 localised around the granulomas to allow their detection.

513

Using the murine i.v. injection model we showed a strong IFN $\gamma$ response was evident at 2-weeks post injection in all mice across the different groups analysed. At this same time point, a significant decrease in lung granuloma size was observed in all the mice injected with KI eggs. However, an enhanced Th2 response was evident only in mice injected with X5-KI eggs indicating the size of granulomas may keep increasing, driven by the climbing Th2 response around the X5-KI eggs, but this needs further investigation.

The circumoval granulomas represent an accumulation of host immune cells including macrophages, eosinophils and neutrophils, around schistosome eggs [67]. The host immune cells act to protect the surrounding host tissue from toxins produced by the egg, resulting in a physical barrier and the sequestering of these secreted egg products [68]; yet these cells are ineffective at clearing infection [69]. To date, the underlying mechanisms of granuloma formation remain poorly understood, and our current knowledge is based on in vitro studies using cells that have not been obtained from granulomas, thereby hindering an improved understanding of the dual functions of immune killing and healing of granulomas generated by schistosome eggs [69]. Granuloma formation is driven by chemokines released by $\mathrm{T}$ lymphocytes and resident liver cells, including HSC, which attract migrating immune cells to the site of egg deposition [70]. Inhibition of HSC activation can lead to decreased collagen production and fibrosis formation $[30,71]$, features also evident in this current study. We found the growth of HSCs (LX-2 cells) was significantly inhibited when the cells were incubated with SEA from AChE-KI eggs compared with SEA extracted from unmutated eggs. Given activated HSCs are recruited to developing granulomas [30], a possible explanation is that the inactivation or inhibition of HSCs by the SEA of AChE-KI eggs may have resulted in the migration of fewer immune cells (macrophages, eosinophils and neutrophils) and a decline in the lungs in the expression of collagen type 1- $\alpha 1$ (COL1 $\alpha 1$ ), a marker of collagen deposition [72], around the granuloma. The combination of these features in vivo might directly lead to the formation of small granulomas. Our results are supported by previous studies showing paeoniflorin (a monoterpene glycoside), which was shown to down-regulate the activity of AChE in rats [73], reduced fibrosis in mice infected with schistosomes as well as inhibiting HSC proliferation and collagen synthesis [74]. However, whether there are key differences between the way lung and hepatic granulomas develop remains unclear. Further investigation is required to improve our understanding of the modulation of granuloma formation induced by schistosome eggs entrapped in lungs including the dynamic changes in the host response correlating with secreted cytokines and development and subsequent resolution of the granuloma.

\section{Concluding comments}


Establishment of the CRISPR/Cas9-mediated KI system in S. mansoni makes it feasible to generate specific loss-of-function modifications in this parasitic flatworm. Notwithstanding the progress presented here, and in earlier reports on CRISPR/Cas9 activity in S. mansoni [15, 16], future improvement in the efficiency of gene editing can be anticipated from additional modifications and refinements of the methodology. This genome editing approach can be readily extended to the study of other schistosome genes and to those of other trematode species thereby paving the way for high-throughput functional analysis of flatworm genes generally.

\section{Methods}

\section{Maintenance of $S$. mansoni}

All experiments were approved by the Animal Ethics Committee of the QIMR Berghofer Medical Research Institute and performed in quarantine-approved facilities. The study was conducted according to the guidelines of the National Health and Medical Research Council of Australia, as published in the Australian Code of Practice for the Care and Use of Animals for Scientific Purposes, 7th edition, 2004 (www.nhmrc.gov.au). All work related to live S. mansoni life cycle stages was performed in quarantine-accredited premises.

Swiss mice (females, 6 weeks old) were infected with 100 S. mansoni cercariae. Seven weeks post-infection mice were euthanised and adult worms were obtained by portal perfusion with $37^{\circ} \mathrm{C}$ DMEM medium (Invitrogen, Carlsbad, USA). Adult worms were incubated in complete schistosome medium (CSM) containing DMEM medium, supplemented with $10 \%$ (v/v) heatinactivated fetal calf serum, $100 \mathrm{IU} / \mathrm{ml}$ penicillin and $100 \mu \mathrm{g} / \mathrm{ml}$ streptomycin, at $37^{\circ} \mathrm{C}$ in an atmosphere of 5\% CO2 in air overnight. Mouse livers were removed at necropsy and eggs were isolated and purified as described [75]. The eggs were cultured in the CSM at $37^{\circ} \mathrm{C}$ under $5 \%$ $\mathrm{CO} 2$ in air. Soluble worm antigen preparation (SWAP) and soluble egg antigen (SEA) were prepared from adult worms and eggs as described [76, 77].

\section{Guide RNA target selection and reconstruction of GeneArt CRISPR Nuclease Vector} Single guide RNA (gRNA) target sequences ( $<15 \mathrm{bp}$ from the desired edit) were selected using the web-based tools available for CRISPR design prediction programs: http://bioinfogp.cnb.csic.es/tools/breakingcas/ [78], and (2) Benchling Referral Program (https://benchling.com) to predict cleavage sites for the Streptococcus pyogenes Cas9 nuclease within the genome of $S$. mansoni. The gRNA targeted exon 5 and 7 of the AChE (Gene ID Smp_154600, www.genedb.org), residues 722-741 (named X5), 1738-1757 (named X7), respectively, adjacent to the protospacer adjacent motif, AGG (Fig 1A). The Smp_154600 includes 9 exons interspersed with 8 introns (57.78kb) (Fig 1A). A double stranded DNA sequence complementary to the gRNA was inserted into GeneArt CRISPR Nuclease Vector (Life technologies, Carlsbad, USA) according to the manufacturer's instructions, which encodes Cas9 from Streptococcus pyogenes driven by the human cytomegalovirus (CMV) immediate 
early promoter and sgRNA driven by the human U6 promoter. The sequences of the two gRNA of Smp_154600 are shown: X5-gRNA, CACCAGGTAATATGGGTCTC (Fig 1B); X7-gRNA, TGGGCTAACTTTGCACGCAC (Fig 1C) (GACCAGGATGGGCACCACCC). Those gRNAs were synthesized by Integrated DNA Technologies (Singapore).

Two single-stranded oligodeoxynucleotides (ssODN) targeting AChEX5 (X5ssODN), AChEX7 (X7ssODN) were synthesized by Integrated DNA Technologies (Singapore). The sequences of the three ssODNs are shown:

1) $\mathrm{X} 5 \mathrm{ss} O \mathrm{DN}$, with homology arms of $50 \mathrm{nt}$ each in length at the 3' (position 738-787 nt) and 5' (688-737 nt) flanks and a small transgene (5'- TAAGTGACTAGGTAACTGAGTAGC-3', encoding stop codons (six) in all reading frames) (Fig 1B);

2) $X 7 \mathrm{ssODN}$, with homology arms of $50 \mathrm{nt}$ each in length at the $3^{\prime}$ (position 1754-1803nt) and 5'(1704-1753nt) flanks and a small transgene (5'- TAAGTGACTAGGTAACTGAGTAGC-3', encoding stop codons (six) in all reading frames) (Fig 1C);

\section{Transfection of adult $S$. mansoni and eggs with a CRISPR Vector with/without ssODN}

608 Pools of 10,000 of eggs or 10 pairs of adult $S$. mansoni were subjected to transfection by electroporation in $100 \mu$ l Opti-MEM containing: 1) Medium only (Medium); 2) X5-gRNA and X5ssODN (3 $\mu \mathrm{g}$ for each) (X5-KI); 3) X5-gRNA (3 $\mu \mathrm{g})$ (X5); 4) X7-gRNA and X7ssODN (3 $\mu \mathrm{g}$ for each) (X7-KI); 5) X7-gRNA (3 $\mu \mathrm{g})(X 7)$; 6) X5-gRNA, X5ssODN and X7-gRNA, X7ssODN (3 $\mu \mathrm{g}$ for each) (X5+X7-KI); 7) X5-gRNA and X7-gRNA (3 $\mu \mathrm{g}$ for each) (X5+X7); 8) GeneArt ${ }^{\circledR}$ CRISPR Nuclease Vector reconstructed with negative gRNA (3 $\left.\mu \mathrm{g}\right)$ (CON); 9) X5ssODN $(3 \mu \mathrm{g})$; 10) X7ssODN $(3 \mu \mathrm{g})$. The mixture was pipetted into a $4 \mathrm{~mm}$ pre-chilled electroporation cuvette and subjected to a square wave with a single $20 \mathrm{~ms}$ impulse at $125 \mathrm{v}$ [79] (Gene Pulser Xcell Electroporator, Bio-Rad, USA), and subsequently maintained at $37^{\circ} \mathrm{C}, 5 \%$ $\mathrm{CO} 2$ in air for 2 days. The eggs and adults were collected on day 2 post electroporation and genomic DNA was extracted from eggs and adults using E.Z.N.A. SQ Tissue DNA Kit (OMEGA bio-tek, Norcross ,USA).

The five controls included parasites subjected to electroporation in the presence of medium only (medium), GeneArt ${ }^{\circledR}$ CRISPR Vector reconstructed with negative gRNA (CON), ssODN only (X5ssODN and X7ssODN). Wide type parasites were cultured without electroporation (WT).

626 Targeting gRNA-AChEX5, PCR assays were performed on each genomic DNA samples 627 extracted from eggs and adults using distinct primer pairs (S1 Table). The primer pairs CF+R4 628 and F2+R4, which amplify locations 22,607-23,411 nt and 22,982-23,411 nt of Smp_154600, 
629 respectively, served as a positive control for the presence of genomic DNA with the 630 Smp_154600 of AChE. The other primer pairs CF+R and F2+R shared one reverse primer (R) 631 complementary to the knock-in 124 nt transgene with two forward primers, CF, F2 at positions 632 22,607-22,626 and 22,982-23,006 nt, respectively, bind complementary to two sites of 633 Smp_154600 targeting site gRNA-AChEX5 DSB (Fig 1B).

Targeting gRNA-AChEX7, PCR assays were carried out on genomic DNA samples extracted from eggs and adults using distinct primer pairs ( $\mathrm{S} 1 \mathrm{Table}$ ). The primer pairs $\mathrm{F} 1+\mathrm{R} 1$ and F1+R6, to amplify locations 37,932-38,518 nt and 37,932-38,403 nt of Smp_154600, respectively, were used as a positive control for the presence of genomic DNA with the Smp_154600. The other primer pairs F+R1 and F+R6 shared one forward primer (F) complementary to the knock-in 124 nt transgene with two reverse primers, R1, R6 at positions 38,499-38,518 and 38,384-38,403 nt, respectively (Fig 2A).

The PCR mix included $10 \mu \mathrm{l}$ Green GoTaq DNA polymerase mix (Promega, Madison, USA) with $200 \mathrm{nM}$ of each primer and $10 \mathrm{ng}$ genomic DNA. Thermal cycling conditions involved denaturation at $95^{\circ} \mathrm{C}, 3 \mathrm{~min}$ followed by 30 cycles of $94^{\circ} \mathrm{C}, 30 \mathrm{sec}, 60^{\circ} \mathrm{C}, 30 \mathrm{sec}$ and $72^{\circ} \mathrm{C}, 30$ sec and a final extension at $72^{\circ} \mathrm{C}$ for $5 \mathrm{~min}$. Amplicons were visualized following agarose gel electrophoresis (1.2\% agarose/TAE), and those of the expected sizes were extracted and purified from gels for sequencing to confirm the presence and knock-out/in of the transgene. Sanger sequencing analysis of the KI amplicons was used to confirm the insertion of the transgene into the AChE locus at X5 and X7 at the predicted cleavage sites.

Illumina sequencing

653 Pooled egg genomic DNA samples from 11 experimental groups of AChEX5 or X7 with/without 654 ssODN were used as the template to amplify the on-target DNA fragment using MiSeq primers 655 (including paired primers F2+R, F2+R4, F+R6, F1+R6) (Fig 1D) with NEBNext Ultra II Q5 656 Master Mix (New England Biolabs, Ipswich, USA). PCR reactions were performed with $60 \mathrm{ng}$ 657 DNA samples from different experimental groups in $50 \mu 1$ reaction mix using the PCR program $65898^{\circ} \mathrm{C}$ for $30 \mathrm{sec}$ of denaturation followed by 35 cycles of $98^{\circ} \mathrm{C}$ for $10 \mathrm{sec}, 65^{\circ} \mathrm{C}$ for $30 \mathrm{sec}, 72^{\circ} \mathrm{C}$ 659 for $30 \mathrm{sec}$ and final extension at $72^{\circ} \mathrm{C}$ for $2 \mathrm{~min}$. The amplicons of expected sizes were purified 660 by using QIAquick Gel Extraction kit (Qiagen, Hilden, Germany). Amplicons generated from 26614 different PCR reactions from each group were pooled, and 100ng of amplicons from each 662 sample were used to construct the uniquely indexed paired-end read libraries (BGI, Hong Kong). 663 These libraries were pooled and the pooled library was quantified using a bioanalyzer (Agilent 664 2100, Agilent Technologies, Santa Clara, USA) combined with StepOnePlus Real-Time PCR 665 System (Applied Biosystems ${ }^{\mathrm{TM}}$, Carlsbad, USA) to measure the adapters before sequencing. The 666 qualified libraries were sequenced on MiSeq using paired-end 300bp reads (Illumina, San Diego, 667 USA). In total, 22 multiplexed samples (S2 Table) were run on MiSeq. 
Amplicon NGS libraries are available at the European Nucleotide Archive under the study accession number EGAS00001004455.

\section{CRISPResso2 analysis}

672 To detect the amount of CRISPR/Cas9-mediated editing in S. mansoni eggs, raw fastq files from

673 individual samples were run through the command line version of CRISPResso2 (v2.0.30) [28].

674 The following parameters were used for CRISPResso2 analysis: "fastq_r1", "fastq_r2",

675 "amplicon_seq", "guide_seq", "expected_hdr_amplicon_seq", "trimmomatic_options_string" to 676 provide Illumina adapters, "max_paired_end_reads_overlap" of 200, "exclude_bp_from_left"

677 equal to the length of the respective forward primer +1 , and "exclude_bp_from_right" equal to 678 the length of the respective reverse primer +1 . The remaining parameters were kept at default 679 values. Notably, the "window_around_sgrna" parameter was kept at 1 to ensure PCR and/or 680 sequencing artefacts were not counted as false positive NHEJ insertions, deletions, or 681 substitutions. Separately, we tested increasing values of the "window_around_sgrna" parameter $682(20,100$, and 0 , where 0 disables the argument to consider the entire length of the amplicon) to 683 determine its effect on the occurrence of NHEJ and HDR events.

685 To confirm potential HDR reads reported by CRISPResso2, we parsed the 686 "Alleles_frequency_table.txt" output file using a custom python script. First, to ensure each read 687 matched the respective forward and reverse primers, the $5^{\prime}$ end of the read (i.e. start of the read to 688 the length of the forward primer) was compared to the sequence to the forward primer, and the 3' 689 end of the read (i.e. end of the read to the length of the reverse primer) was compared to the 690 reverse complement of the reverse primer, and allowed for $1 \mathrm{bp}$ mismatch using the 'levenshtein` 691 function within the 'distance' python package. These filtered reads were then used to determine 692 the editing frequencies of NHEJ and HDR. The HDR reads reported by CRISPResso2 were then 693 confirmed using fasta36 (v3.8; https://github.com/wrpearson/fasta36), where an HDR read was 694 considered 'confirmed' if it had $>90 \%$ identity to the expected knock-in sequence.

\section{AChE activity assays}

697 Cultured adult worms and eggs were collected on day 2 after electroporation for protein 698 extraction of soluble worm antigen preparation (SWAP) [76] and soluble egg antigen (SEA) [32] 699 as described. The AChE activity in these SWAP $(7.5 \mu \mathrm{g} / \mathrm{mL})$ and SEA $(0.45 \mu \mathrm{g} / \mathrm{mL})$ 700 preparations were measured using the Amplex Red Acetylcholine/Acetylcholinesterase Assay 701 Kit (Invitrogen) according to the manufacturer's instructions.

\section{In vitro scratch wound assays}

704 In vitro scratch wound assays were carried out to determine the effect of SEA extracted from 705 control and CRISPR/Cas9 mutated eggs [80, 81]. HSC cells (LX-2) were cultured in completed 706 medium containing high glucose EMDM (Invitrogen), 2\% fetal bovine serum (FBS), 1\% 707 glutamax and 1\% Pen/strep. Cells were grown to create a confluent monolayer in 96-well plates. 
Then the monolayers were scraped in a straight line to create a "scratch" using the Wound Maker-IncuCyte ZOOM-Image Lock Plate system (Essen Bioscience, Michigan, USA) and washed once with the culture medium. Cells were continued to culture in the fresh completed medium containing $30 \mu \mathrm{g} / \mathrm{ml}$ SEA. Residual endotoxin was assessed by using an Endotoxin Standards kit (Lonza, Anaheim, USA) to ensure there was no LPS contamination in the SEA extracted from control and AChE-KI eggs). The growth of cells was monitored by IncuCyte Zoom for 3 days. Images were acquired for each well at 3 hours interval by an in-built phase contrast microscope. The area of the scratch was measured using ImageJ (image processing program, Java). The total area $\left(\mu \mathrm{m}^{2}\right)$ was obtained at every time point until would closure, and triplicate measures were compared to calculate an average closure rate per group (area of original wound/time to closure in hours). All data were then statistically analysed using GraphPad Prism Software V7.

\section{Pathological changes and immune responses induced by intravenous injection of AChE-KI} eggs in mice

Mutated and unmutated egg batches were intravenously (i.v.) injected into mice under sterile conditions as described [82]. AChE-KI eggs were cultured for 2 days following electroporation with mixtures of X5-KI; X7-KI; X5+X7-KI, respectively. Briefly, 1,000 eggs in $100 \mu 1$ of sterile PBS were injected into the lateral tail vein of female Swiss mice (7-8 weeks of age). Mice in control groups were injected with PBS (naive), untreated eggs (WT) or with eggs electroporated with negative control GeneArt vector (Con). All mice were euthanised 2 weeks after injection. This experiment was repeated twice with each group comprising 5 mice.

\section{Measurement of lung granuloma size in mice} these samples were prepared and stained with Haematoxylin and Eosin (H\&E). Slides were digitized using an Aperio Slide Scanner (Aperio Technologies, Vista, USA). The degree of lung pathology was quantified by measurement of the area density of granulomatous lesions using Aperio Image Scope v11.1.2.760 software (Leica Biosystems Imaging, Buffalo Grove, USA), and was estimated from the area of the granulomas divided by the total area of the liver tissue in the image.

\section{Immune responses induced by intravenous injection of AChE-KI eggs} injection, and sera were prepared and stored at $-80^{\circ} \mathrm{C}$. The total $\operatorname{IgE}$ level of individual sera was measured using an IgE mouse ELISA kit (Thermo Fisher Scientific, Waltham, USA), according to the manufacturer's instructions.

2) Cytokine analysis. The spleen and lung of each mouse ( $n=5$ per group) were collected and splenocytes [83] and lung cells [84] were isolated as described. Briefly, the individual lung was 
finely chopped and digested in $1 \mathrm{~mL}$ of lung digestion buffer, containing $4 \mathrm{mg} / \mathrm{mL}$ collagenase D (Sigma-Aldrich, St. Louis, USA), 10\% FCS (Fetal Calf Serum, Invitrogen) $0.5 \mathrm{mg} / \mathrm{mL}$ DNase (Promega) in IMDM (Iscoves Modified Dulbecco's Medium, Invitrogen), for $1 \mathrm{hr}$ at $37^{\circ} \mathrm{C}$ and 150 RPM in a shaking incubator. The digested lung was then mechanically disrupted through a syringe followed by filtering through a $70 \mu \mathrm{m}$ cell strainer. The individual spleen was pressed through a $70 \mu \mathrm{m}$ cell strainer. All the spleen and lung samples were then lysed using RBC lysis buffer (Sigma-Aldrich, Missouri, USA) to remove red blood cells. Following repeated washing and centrifugation steps, the cells were resuspended in IMDM containing $10 \%$ (v/v) fetal bovine serum, $100 \mu \mathrm{g} / \mathrm{ml}$ Streptomycin, 100 Units/ml Penicillin and $0.05 \mathrm{mM}$ 2-Mercaptoethanol. Cell counts were determined by trypan blue exclusion, and then cells were seeded into 96-well plates $\left(5 \times 10^{5}\right.$ cells/well). The cells were incubated in the presence of SEA ( $2 \mu \mathrm{g} / \mathrm{well}$, extracted from S. mansoni liver eggs) for 72 hours at $37^{\circ} \mathrm{C}$. Stimulation with ConA $(0.1 \mu \mathrm{g} /$ well $)$ was performed as a positive control and non-stimulated splenocytes or lung cells were used as a negative control. Each cell culture supernatant $(50 \mu \mathrm{l} /$ well) was harvested and secreted cytokines (IFN $\gamma$, TNF $\alpha$, IL-2, 4, 5, 6, 10, 13) were assayed using LEGEND plex Mouse Th1/Th2 panel kits (BioLegend, San Diego, USA) according to the manufacturer's instructions.

3) Real time PCR. A small loop of lung was cut individually from each mouse injected i.v. with AChE-KI eggs. Total RNA was extracted from the lung tissue using Trizol (Invitrogen) and an RNeasy Mini Kit (Qiagen) [85] followed by DNase (Promega) digestion; cDNA was synthesised as described [86]. Real-time PCR was performed and analysed using the ABI ViiA ${ }^{\mathrm{TM}} 7$ real time PCR system (Thermo Fisher Scientific). TaqMan Gene Expression Assays (ThermoFisher) for (resistin-like molecules, Mm00445109) were employed to detect potential disturbances in Th2 polarization. HPRT (hypoxanthine guanine phosphoribosyl transferase) was used as a reference gene to normalise all data generated [87].

The expression level of COL1 $\alpha 1$ in lung tissue of mice injected with mutated eggs was determined by qPCR quantification using SYBR Green master mix (Applied Biosystems, Foster City, USA) on a Corbett Rotor Gene 6000 (Corbett Life Sciences, Uithoorn, Netherlands). cDNA obtained from lung tissue of mice in treated and untreated groups was used as template and primer sequences for COL1 $\alpha 1$ were as previously reported $[88,89]$. Data were analysed by

\section{Immune responses induced by intestinal injection of AChE-KI eggs into mouse lymph nodes}

784 importing the standard curve to each run using Rotor-Gene 6000 Series software (version 1.7).

1) Egg intestinal injection surgery. Laparotomy surgery was performed as described [36]. In brief, mice were anaesthetised using Isoflurane (Abbot Animal Health), a small incision into the skin and the muscle layer of the animal's midline was made using a scalpel and extended using scissors. The intestine was carefully displayed onto a surgical cloth using cotton buds and moistened. Mutated or unmutated $S$. mansoni eggs were resuspended at 4,000 eggs/50 $\mu 1$ PBS 
and 4,000 eggs were injected into the subserosal layer of the ileum at two locations using MicroFine Plus Hypodermic Syringes (29G x 12.7mm; BD Bioscience). After successful egg injection, the intestines were replaced into the body cavity and the muscle and skin incisions closed using 6.0 Vicryl absorbable sutures (Johnson and Johnson). The analgesics Buprenorphine $(0.1 \mathrm{mg} / \mathrm{kg}$; Vetergesic, Reckitt Benckiser Healthcare) and Carprofen (5 mg/kg; Rimadyl, Pfizer) were administered subcutaneously into the flank and mice were closely monitored post-surgery to ensure full recovery from the anaesthesia and monitored on a daily basis thereafter. This experiment was repeated twice, with each group comprising 4-6 mice.

2) Cell isolation and in vitro stimulation. Mice were scarified on day 5 post egg injection and the ileum draining mesenteric lymph nodes were isolated as described [36]. Single cell suspensions were obtained by disrupting each lymph node with a $70 \mu \mathrm{m}$ cell strainer (BD Bioscience). In each experiment, cells were split to perform flow cytometric analysis and to set up re-stimulation cultures from the same sample. $2 \times 10^{6}$ cells were incubated in RPMI 1640 (Life Technologies), supplemented with $2.5 \mathrm{ng} / \mathrm{ml}$ PMA (Sigma-Aldrich), $1 \mathrm{mg} / \mathrm{ml}$ ionomycin (Invitrogen), 0.5\% GolgiStop (BD Bioscience, Franklin, USA) and 10\% FCS for four hours at $37{ }^{\circ} \mathrm{C}$, after which live/dead staining was performed and cell surface markers were stained. Cells were fixed and permeabilized using the eBioscience Foxp3/Transcription Factor Staining Buffer Set (eBioscience, San Diego, USA) and intracellular staining was performed following the manufacturer's instructions. For re-stimulation cultures, 1 x $10^{6}$ MLN cells were cultured in X-vivo 15 medium (Lonza) supplemented with 1\% L-glutamine (Invitrogen), 0.1\% 2mercaptoethanol (Sigma-Aldrich) and $7.5 \mu \mathrm{g} / \mathrm{ml}$ SEA in round bottom 96-well plates (Corning) at $37^{\circ} \mathrm{C}$ and $5 \% \mathrm{CO}_{2}$. Supernatants were collected after three days and cytokine levels were determined using IL-4, IL-13 and IFN- $\gamma$ "ready-set-go" ELISA kits or paired antibodies

\section{2 (eBioscience).}

3) Antibodies for flow cytometric analysis. The following combination of fluorescently labelled primary antibodies against cell surface markers, intracellular cytokines and transcription factors were used: anti-CD3 (17A2), anti-CD4 (clones GK1.5), anti-CD8a (53-6.7), anti-CD44 (IM7), anti-CD45R/B220 (RA3-6B2) and anti-IL4 (11B11) from Biolegend, and anti-IFN- $\gamma$ (XMG1.2) and anti-GATA3 (TWAJ) from eBioscience. Cells were analysed using a BD LSRFortessa flow cytometer running FACSDiva Software (BD Bioscience) and analysed using FlowJo Software (Tree Star).

823 All data are presented as the mean \pm SE. Differences between groups were assessed for statistical 824 significance using One-way ANOVA; Tukey post hoc test or Kruskal-Wallis were used for 825 comparisons involving > 2 groups compared with the control. GraphPad Prism software (Version 826 7, GraphPad Software, La Jolla, CA, USA) was used for all statistical analyses. $P$ values $\leq 0.05$ 
bioRxiv preprint doi: https://doi.org/10.1101/2020.07.08.190694; this version posted July 9, 2020. The copyright holder for this preprint (which was not certified by peer review) is the author/funder. All rights reserved. No reuse allowed without permission.

827 were considered to be statistically significant. ${ }^{*} p<0.05, * * p<0.01, * * * p<0.001$; not significant 828 (ns).

829 
Fig 1. CRISPR/Cas9-mediated editing of the acetylcholinesterase (AChE) locus.
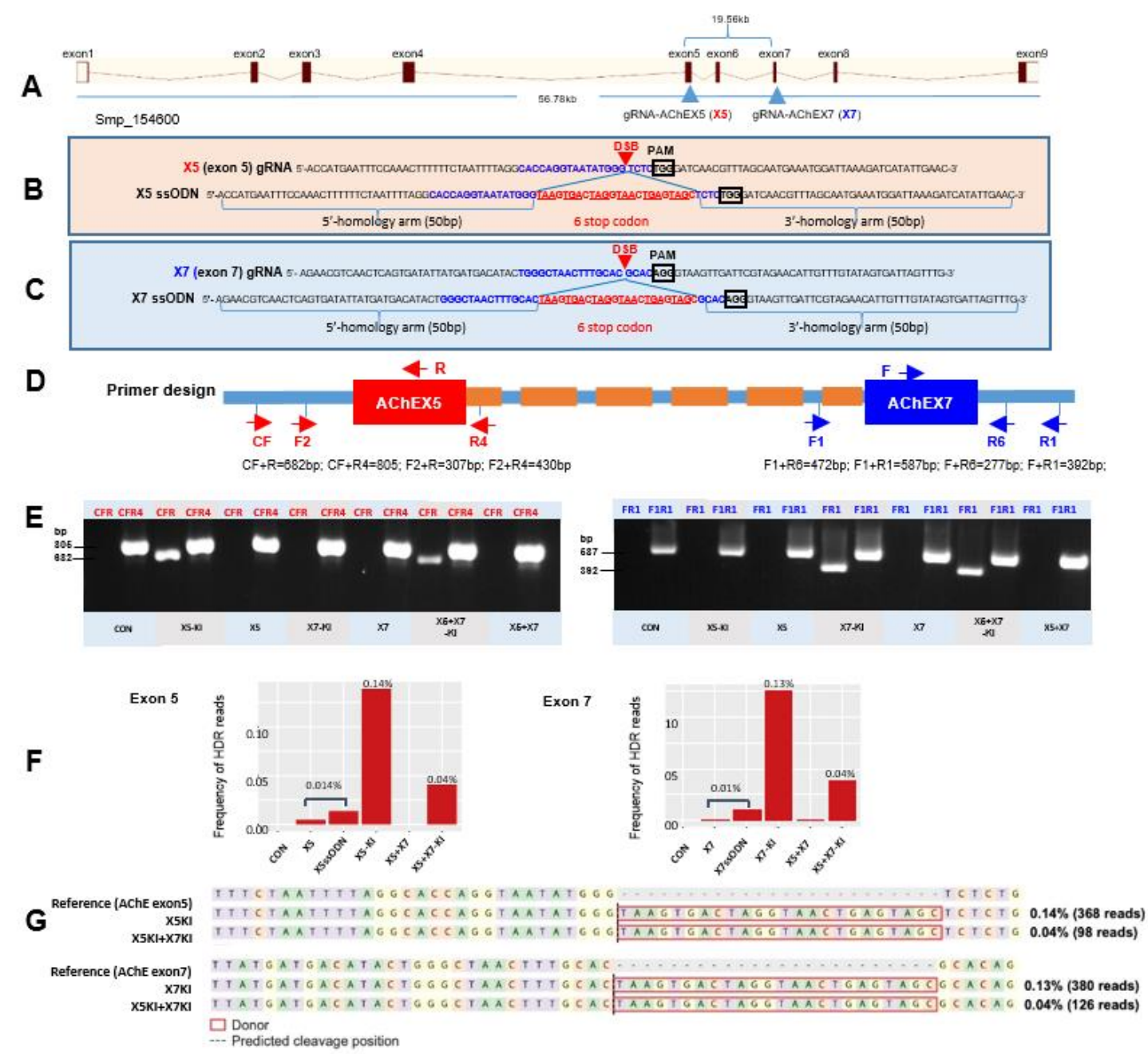

(A) Schematic diagram of the AChE (Smp_154600) gene in S. mansoni. Two guide RNAs (gRNAs) were designed to target AChE: X5 targeting exon 5 and X7 targeting exon 7. Partial nucleotide sequence of (B) AChE exon 5 and (C) exon 7 indicating the respective gRNA sequence, target site, predicted double stranded break (DSB), protospacer adjacent motif (PAM), and sequence of the single-stranded oligodeoxynucleotide ( $s \mathrm{ODN}$ ) donor template. Homology arms of $50 \mathrm{nt}$ flank a central $24 \mathrm{nt}$ six-stop-codon transgene. (D) Schematic diagram of AChE indicating positions and directionality of primer binding sites (arrows). Primers in red target $\mathrm{AChE}$ exon 5 (CF, F2, R, R4) and primers in blue target AChE exon 7 (F1, F, R6, R1). The control PCR amplicons were generated using the $\mathrm{CF}+\mathrm{R} 4$ primers (red arrows) targeting the fragment around gRNA-X5, and F1+R1 primers (blue arrows) targeting the fragment around gRNA-X7. (E) PCR products demonstrating CRISPR/Cas9-mediated editing in exon 5 (left panel) and 7 (right panel) of the AChE gene. Genomic DNA extracted from eggs treated with $\mathrm{CON}, \mathrm{X} 5-\mathrm{KI}, \mathrm{X} 5, \mathrm{X} 7-\mathrm{KI}, \mathrm{X} 7, \mathrm{X} 5+\mathrm{X} 7-\mathrm{KI}$ and $\mathrm{X} 5+\mathrm{X} 7$ were used as the PCR template. Evidence 
847 of X5-KI and X7-KI revealed by amplicons of the expected sizes in lanes CFR (682 bp) and FR1

848 (392 bp), respectively, spanned the mutated site in the genomic DNAs pooled from schistosome 849 eggs, including positive controls flanking the insert site of gRNA-X5 (CFR4, 805 bp) and the 850 insert site of gRNA-X7 (F1R1, $587 \mathrm{bp})$. The control DNA result shown in this gel was isolated 851 from eggs electroporated with negative control vector (CON) only. (F) Frequency of homology 852 directed repair (HDR) at AChE exon 5 (left panel) and exon 7 (right panel) in amplicon deep 853 sequencing data. Amplicons at exon 5 were generated using primer pair F2+R4, and amplicons at 854 exon 7 were generated using primer pair F1+R6 as indicated in (D). HDR reads were detected 855 using CRISPResso2 and confirmed using fasta36. The number of confirmed HDR reads are 856 shown, expressed as a percentage of aligned reads. (G) Sequence alignments and HDR 857 frequencies for amplicon deep sequencing data. Nucleotide sequences for AChE exon 5 (upper 858 panel) and exon 7 (lower panel), extending $30 \mathrm{bp}$ either side of the respective predicted cleavage 859 position, are shown. At both loci, the presence of a 24 nt transgene (red outline) was confirmed. 860 HDR frequencies, expressed as a percentage of aligned reads, are shown to the right of each KI 861 group. Plots were based on default output from CRISPResso2. 
Fig 2. Effects of AChE-KI eggs of S. mansoni on AChE enzyme activity, LX-2 cell growth

866

867

868

869

870

871

872

873

874

875

876

877

878

879

880

881
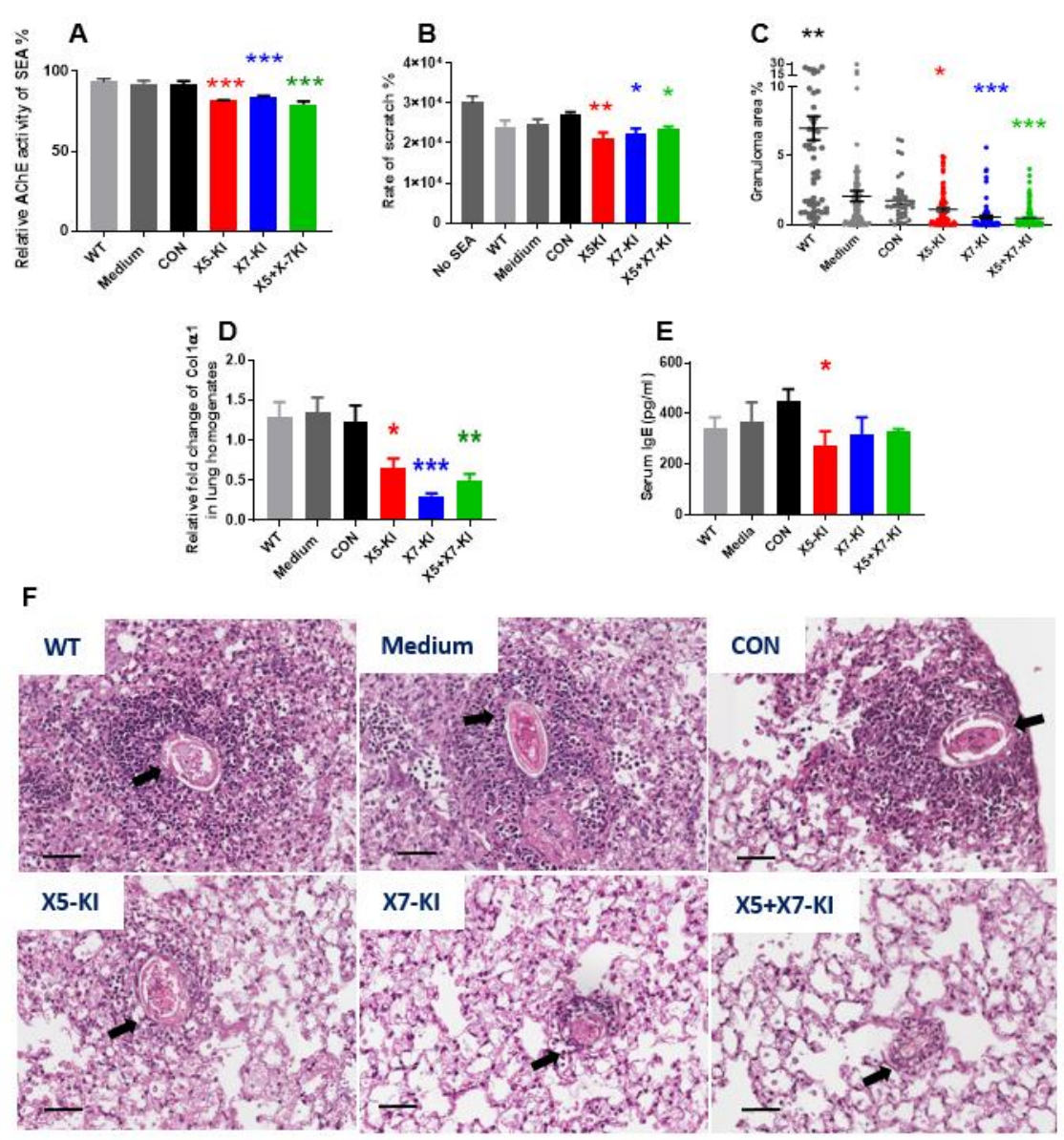

(A) Diminished AChE activity of SEA extracted from $S$. mansoni eggs after incubation with medium, CON, X5-KI, X5, X7-KI, X7 and X5-KI+X7-KI, respectively. SEA extracted from Wild type (WT) eggs were used as positive control. (B) Monitoring LX-2 cell growth on day 3 after being incubated with mutated/unmutated SEA. The scratch wound assay using the IncuCyte system measured the migration of LX-2 cells. The total area $\left(\mu \mathrm{m}^{2}\right)$ was obtained at every time point (from day 0 to day 3) until wound closure, and triplicate measures were compared to calculate an average closure rate per group (area of original wound/time to closure in hours). No SEA: cells cultured in medium without SEA. (C) Effect of AChE-KI eggs on granuloma formation in the lungs of mice i.v. injected with eggs treated with X5-KI, X7-KI and X5-KI+X7KI, respectively. A control group of mice received untreated WT eggs or CON eggs. All mice were euthanized 2 weeks after injection. Granuloma sizes were determined as the ratio of the granuloma area to the egg area. The scatter plot shows the mean \pm SE. of the data pool representing all granulomas from all lungs from each experimental group (WT: $n=60 ; \mathrm{CON}=40$; X5-KI: $n=88$; X7-KI: $n=72 ; X 5+X 7-K I: ~ n=120$ ); each group included 10 mice. (D) Transcription level of collagen type 1 (alpha 1-COL1 $\alpha 1$ ) in S. mansoni eggs in different experimental groups. 
882 HPRT (hypoxanthine guanine phosphoribosyl transferase) was used as a reference gene to 883 normalise data [87]. (E) Total serum IgE levels in mice two weeks following i.v. injection of WT 884 eggs of $S$. mansoni and eggs treated with medium, CON, X5-KI and X7-KI, X5+X7-KI. For 885 panels A-E, each experiment was performed in duplicate and all data are presented as the mean \pm 886 SE. One-way ANOVA analysis was used to establish statistical significance compared with the 887 CON control: $*=p$ value $\leq 0.05, * *=p$ value $\leq 0.001, * * *=p$ value $\leq 0.0001$. (F) Granuloma size 888 in lung sections from mice i.v. injected with eggs of $S$. mansoni in different groups, examined 889 microscopically following H \& E staining. Scale bars, 50 $\mathrm{m}$. WT, wild type parasites; Medium, 890 parasites electroporated with medium; CON, parasites treated with negative control vector. 

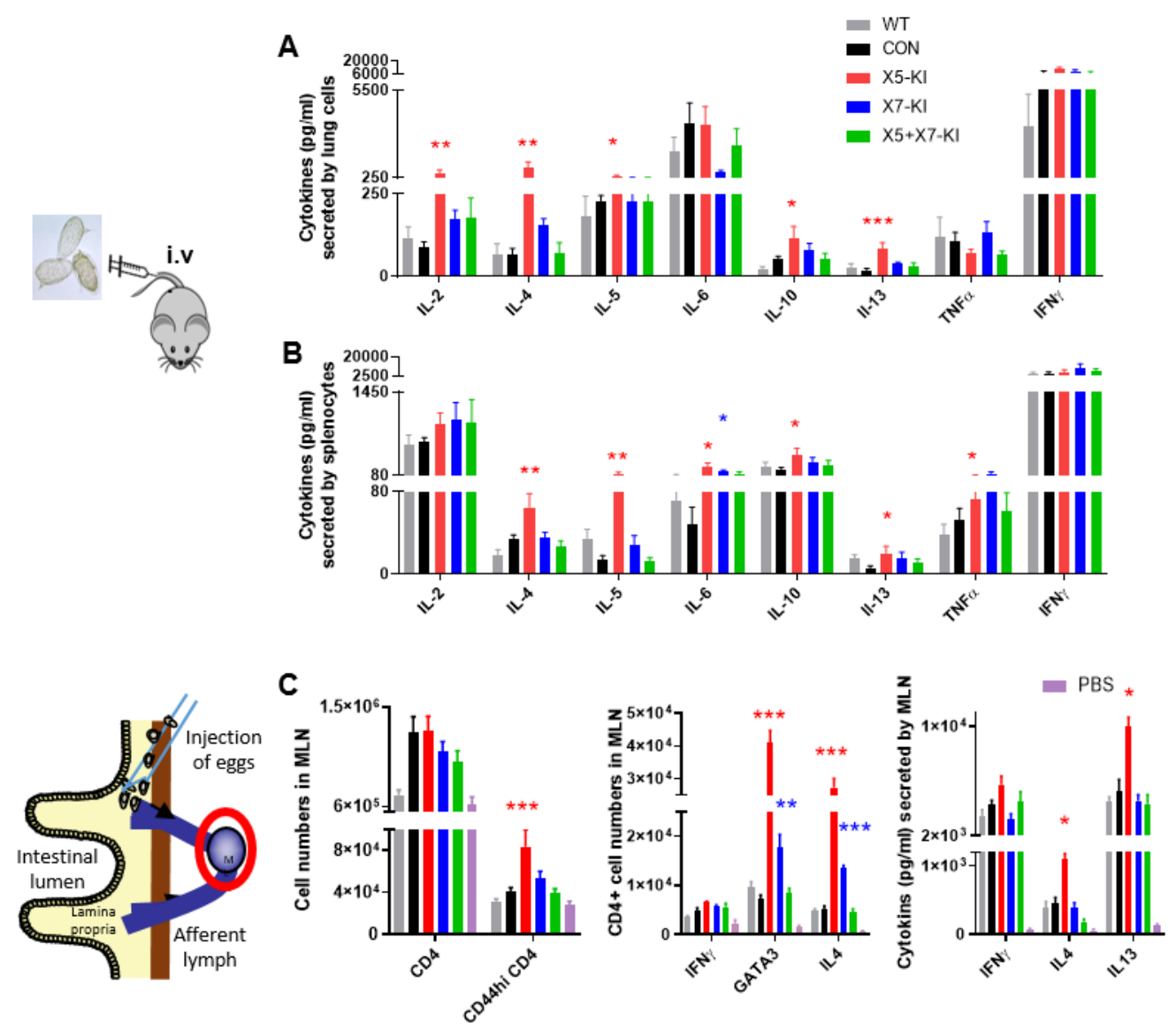

895

896

897

898

899

900

901

902

903

904

905

906

907

908

909
To test cytokine secretion of immune cells derived from lung and spleen, lung cells and splenocytes from mice were analysed at 2 weeks after i.v. injection, via the tail vein, with WT eggs of $S$. mansoni and eggs treated with empty-vector (CON), X5-KI, X7-KI and X5+X7-KI. Cytokines (IFN $\gamma, \mathrm{TNF} \alpha$, IL-2, IL-4, IL-5, IL-6, IL-10 and IL-13) were quantified in culture supernatants of (A) lung cells and (B) splenocytes restimulated with SEA for 72 hours in the different treated and untreated groups. To determine the cytokine profiles of intestinal immune responses induced by AChE-KI eggs, ileum draining mesenteric lymph node (MLN) cells were isolated from mice on day 5 after subserosal injection with WT and CON eggs of $S$. mansoni and eggs treated X5-KI, X7-KI and X5+X7-KI. (C) Left panel, the proportion of $\mathrm{CD}^{+}{ }^{+} \mathrm{T}$ cells, CD44hi CD4 ${ }^{+}$cells; Middle panel, the proportion of GATA3-, IFN $\gamma$ - and IL-4- producing MLN $\mathrm{CD}^{+} \mathrm{T}$ cells isolated from MLN cells for each mouse were determined; right panel, secreted cytokines (IFN $\gamma$, IL-4 and IL-13) were quantified in culture supernatants following 72 hours culture. WT, Wild Type; CON, Control; IL, interleukin; IFN, interferon; TNF $\alpha$, Tumor necrosis factor alpha; PBS, Phosphate-Buffered Saline. Each experiment was performed in duplicate; data 
910 are presented as the mean \pm SE and one-way ANOVA analysis was used to establish statistical significance compared with the $\mathrm{CON}: * p<0.05, * * p<0.01, * * * p<0.001$

912

\section{Supplementary information}

914

S1 Fig. Discrete exon/intron structure of the loci encoding genes Smp_136690 and Smp_125350 (two paralogues of Smp_154600, acetylcholinesterase) in S. mansoni.

S2 Fig. Amino acid alignment of proteins Smp_136690, Smp_125350 and Smp_154600. Red boxes indicate the two conserved subdomains including the carboxylesterase type-B signature 2 (E163-P173 in Smp_154600) and carboxylesterase type-B serine active site (F264-G279 in Smp_154600). Motifs include N-myristoylation sites boxed in purple (G77-L82, G101-Q106, G304-N309, G401-E406, G539-Y544 in Smp_154600). The conserved active catalytic triad site (shown as a red star) is located at E406 in Smp_154600, while the 4 residues (W154, W192, Y208, Y544 in Smp_154600, in red triangles) in the rings of 14 aromatic amino acid residues of S. mansoni AChE, which contribute to the 3D- structure of AChE, are conserved in the appropriate locations in the two molecules.

S3 Fig. (A) PCR products visualized in ethidium bromide-stained agarose gels demonstrating Cas9-catalyzed target site-specific insertional mutagenesis in exon 5 and 7 of the AChE gene. Genomic DNA extracted from adult S. mansoni treated with CON, X5-KI, X5, X7-KI, X7 and $\mathrm{X} 5+\mathrm{X} 7-\mathrm{KI}$, respectively, was used as template for PCR. Evidence for transgene knocked-in into the programmed target site revealed by amplicons of the expected sizes in lanes CFR and FR 1 of 682 and 392 bp, respectively; these span the mutated site in the genomic DNAs pooled from adult $S$. mansoni, including positive controls flanking the insert site of gRNA-X5 (CFR4, 805 $\mathrm{bp})$ and the insert site of gRNA-X7 (F1R1, $587 \mathrm{bp})$. The control DNA was isolated from adult worms electroporated with medium only. (B) AChE activity of soluble worm antigen preparation (SWAP) extracted from adult S. mansoni that had been treated with medium, CON, X5-KI, X5, X7-KI, X7 and X5+X7-KI, respectively. SWAP extracted wild type (WT) adult worms were used as positive control.

S4 Fig. Electroporation of eggs does not induce a significant difference in egg viability. Eggs isolated from the liver of an $S$. mansoni-infected mouse were hatched. (A) The hatching rates were calculated before and after electroporation. Data are presented as the mean $\pm \mathrm{SE}$; t-test analysis showed $p=0.16$. (B) Hatched miracidia visualised under the microscope.(a) mature egg in the process of hatching; (b) miracidium escaping from an egg; (c) empty eggshell after hatching; (d) free-swimming miracidium in medium following hatching; (e) immature egg. 

modified reads. Stacked bar plots of the number of modified reads expressed as a percentage of aligned reads per experimental sample. The CRISPResso2 "-w" window parameter was changed to a (A) value of 1 , (B) value of 20 , (C) value of 100 , and (D) value of 0 (the entire amplicon length) while keeping all other CRISPResso2 parameters constant. The results of each of the four analyses have been separated into two groups: experimental samples amplified using primers targeting AChE Exon 5 (upper panels) and experimental samples amplified using primers targeting AChE Exon 7 (lower panels).

S6 Fig Images of the growth of LX-2 cells on day 0, 1 and day 2 after incubation with soluble egg antigens (SEA) extracted from: (A) wild type (WT) eggs; (B) eggs electroporated with medium; (C) CON eggs; (D) X5-KI eggs; (E) X7-KI eggs and (F) X5+X7-KI eggs. Scale bars, $961300 \mu \mathrm{m}$.

962

S7 Fig. Cytokine mRNA expression observed in the lungs of mice i.v. injected with CRISPR/Cas9 mutated/unmutated eggs. A portion of mouse lung homogenate was used to determine, by qPCR, the expression levels of: (A) IL-4, (B) INFr, (C) RetnlA (resistin-like molecules) and (D) Ctla4. One-way ANOVA analysis was used to establish statistical significance compared with the $\mathrm{CON}: * p<0.05, * * p<0.01, * * * p<0.001$.

S8 Fig. Gating strategy for the assessment of Th1 and Th2 cells in the MLN after subserosal injection with $S$. mansoni eggs. MLNs were harvested 5 days after subserosal egg injection, digested and stimulated with PMA/ionomycin for 4 hours. CD4 T cells were identified by flow cytometry analysis by gating on live single cells that were B220- CD3+ and CD4+. IFN$\gamma+$ CD4+ Th1 cells and GATA3+ and IL-4+ Th2 cells were quantified after the injection of WT $S$. mansoni eggs or eggs treated with negative control vector (CON), X5-KI, X7-KI or X5$\mathrm{KI}+\mathrm{X} 7-\mathrm{KI}$.

S9 Fig. Immunolocalisation showing that the expression of AChE by Schistosoma eggs in liver tissue is distributed throughout the eggs [26] and is highly expressed in mature eggs. Adapted from our previous work [26], we showed that AChE is located in the thin cellular epithelium, the extra-embryonic inner envelope of eggs, and in cells of the inner-most cellular layers of advanced S. japonicum egg granulomas [26]. AChE is highly expressed in the mature egg compared with immature egg, in the former of which the miracidium has developed within the eggshell into a multi-cellular, mobile, ciliated larva comprising organs, tissues, muscles and nerves [26]. Given the high level of conservation (88\% amino acid identity) in protein sequences for AChE in S. japonicum and S. mansoni [25], we assume the distribution of AChE in the eggs

\section{S1 Table. Sequences of guide RNA and primers used for PCR amplification}


S2 Table. Quantification of the modifications in NGS sequence reads using CRISPResso2 software.

\section{Acknowledgements}

B. glabrata snails provided by the NIAID Schistosomiasis Resource Center of the Biomedical Research Institute (Rockville, MD) through NIH-NIAID Contract HHSN272201700014I for distribution through BEI Resources. This work received support from an Australian Infectious Disease Research Centre Seed Grant and a Program Grant from the National Health and Medical Research Council of Australia (APP 1037304) and from a Strategic Award from the Wellcome Trust, award no. 107475/Z/15/Z, entitled the Flatworm Functional Genomics Initiative (KF Hoffmann, PI, PJ Brindley, co-PI).

1002

1003

1004

\section{Author Contributions}

1005

Conceptualization: Hong You, Haran Sivakumaran, Patrick Driguez, Juliet D. French, Wannaporn Ittiprasert, Paul J. Brindley, Donald P. McManus

1006

1007

Data curation: Hong You, Johannes U. Mayer, Rebecca L. Johnston, Lambros T. Koufariotis, Nicola Waddell

Formal analysis: Hong You, Johannes U. Mayer, Rebecca L. Johnston, Olga Kondrashova, Jones, Donald P. McManus

Methodology: Hong You, Johannes U. Mayer, Rebecca L. Johnston, Haran Sivakumaran,

Project administration: Hong You, Malcolm K. Jones, Donald P. McManus

Resources: Hong You, Mary G. Duke, Donald P. McManus

1018

Supervision: Hong You, Paul J. Brindley, Malcolm K. Jones, Donald P. McManus

1019

1020

Validation: Hong You, Johannes U. Mayer, Haran Sivakumaran, Juliet D. French, Nicola Waddell, Paul J. Brindley, Malcolm K. Jones, Donald P. McManus

1021

Writing - original draft: Hong You, Johannes U. Mayer, Rebecca L. Johnston, Olga Kondrashova, Haran Sivakumaran

Writing - review \& editing Hong You, Juliet D. French, Nicola Waddell, Paul J. Brindley, 1024 Malcolm K. Jones, Donald P. McManus

\section{References} Engl J Med. 2002;346(16):1212-20. doi: 10.1056/NEJMra012396. PubMed PMID: 11961151. 
1029 2. Hagan P, Appleton CC, Coles GC, Kusel JR, Tchuem-Tchuente LA. Schistosomiasis 1030 control: keep taking the tablets. Trends Parasitol. 2004;20(2):92-7. PubMed PMID: 14747023.

1031 3. Schistosoma japonicum Genome S, Functional Analysis C. The Schistosoma japonicum 1032 genome reveals features of host-parasite interplay. Nature. 2009;460(7253):345-51. doi: 1033 10.1038/nature08140. PubMed PMID: 19606140; PubMed Central PMCID: PMCPMC3747554.

1034 4. Berriman M, Haas BJ, LoVerde PT, Wilson RA, Dillon GP, Cerqueira GC, et al. The 1035 genome of the blood fluke Schistosoma mansoni. Nature. 2009;460(7253):352-8. doi: 1036 10.1038/nature08160. PubMed PMID: 19606141; PubMed Central PMCID: PMCPMC2756445.

1037 5. Young ND, Jex AR, Li B, Liu S, Yang L, Xiong Z, et al. Whole-genome sequence of 1038 Schistosoma haematobium. Nat Genet. 2012;44(2):221-5. doi: 10.1038/ng.1065. PubMed PMID: 103922246508.

1040 6. Hoffmann KF, Brindley PJ, Berriman M. Medicine. Halting harmful helminths. Science. 1041 2014;346(6206):168-9. doi: 10.1126/science.1261139. PubMed PMID: 25301604.

1042 7. Jiang W, Bikard D, Cox D, Zhang F, Marraffini LA. RNA-guided editing of bacterial 1043 genomes using CRISPR-Cas systems. Nat Biotechnol. 2013;31(3):233-9. doi: 10.1038/nbt.2508. 1044 PubMed PMID: 23360965; PubMed Central PMCID: PMCPMC3748948.

1045 8. Lino CA, Harper JC, Carney JP, Timlin JA. Delivering CRISPR: a review of the 1046 challenges and approaches. Drug Deliv. 2018;25(1):1234-57. doi: 1047 10.1080/10717544.2018.1474964. PubMed PMID: 29801422; PubMed Central PMCID: 1048 PMCPMC6058482.

1049 9. Hefferin ML, Tomkinson AE. Mechanism of DNA double-strand break repair by non1050 homologous end joining. DNA Repair (Amst). 2005;4(6):639-48. doi: 1051 10.1016/j.dnarep.2004.12.005. PubMed PMID: 15907771.

1052 10. Team TA. CRISPR 101: A Desktop Resource 2017.

1053 11. Cho SW, Lee J, Carroll D, Kim JS, Lee J. Heritable gene knockout in Caenorhabditis 1054 elegans by direct injection of Cas9-sgRNA ribonucleoproteins. Genetics. 2013;195(3):1177-80. 1055 doi: 10.1534/genetics.113.155853. PubMed PMID: 23979576; PubMed Central PMCID: 1056 PMCPMC3813847.

1057 12. Friedland AE, Tzur YB, Esvelt KM, Colaiacovo MP, Church GM, Calarco JA. Heritable 1058 genome editing in C. elegans via a CRISPR-Cas9 system. Nat Methods. 2013;10(8):741-3. doi: 1059 10.1038/nmeth.2532. PubMed PMID: 23817069; PubMed Central PMCID: PMCPMC3822328.

1060 13. Zhang WW, Matlashewski G. CRISPR-Cas9-Mediated Genome Editing in Leishmania 1061 donovani. mBio. 2015;6(4):e00861. doi: 10.1128/mBio.00861-15. PubMed PMID: 26199327; 1062 PubMed Central PMCID: PMC4513079.

1063 14. Doudna JA, Charpentier E. Genome editing. The new frontier of genome engineering 1064 with CRISPR-Cas9. Science. 2014;346(6213):1258096. doi: 10.1126/science.1258096. PubMed 1065 PMID: 25430774.

1066 15. Ittiprasert W, Mann VH, Karinshak SE, Coghlan A, Rinaldi G, Sankaranarayanan G, et 1067 al. Programmed genome editing of the omega-1 ribonuclease of the blood fluke, Schistosoma 1068 mansoni. Elife. 2019;8:e41337. 
1069 16. Sankaranarayanan G, Coghlan A, Driguez P, Lotkowska ME, Sanders M, Holroyd N, et 1070 al. Large CRISPR-Cas-induced deletions in the oxamniquine resistance locus of the human parasite Schistosoma mansoni. bioRxiv. 2020.

1072 17. Arunsan P, Ittiprasert W, Smout MJ, Cochran CJ, Mann VH, Chaiyadet S, et al. 1073 Programmed knockout mutation of liver fluke granulin attenuates virulence of infection-induced 1074 hepatobiliary morbidity. Elife. 2019;8:e41463.

1075 18. Robertson AP, Martin RJ. Ion-channels on parasite muscle: pharmacology and 1076 physiology. Invert Neurosci. 2007;7(4):209-17. doi: 10.1007/s10158-007-0059-x. PubMed 1077 PMID: 17999098.

1078 19. Kaminsky R, Gauvry N, Schorderet Weber S, Skripsky T, Bouvier J, Wenger A, et al. 1079 Identification of the amino-acetonitrile derivative monepantel (AAD 1566) as a new anthelmintic drug development candidate. Parasitol Res. 2008;103(4):931-9. doi: 10.1007/s00436-008-10807. PubMed PMID: 18594861; PubMed Central PMCID: PMCPMC2491438.

1082 20. Bueding E, Liu CL, Rogers SH. Inhibition by metrifonate and dichlorvos of 1083 cholinesterases in schistosomes. Br J Pharmacol. 1972;46(3):480-7. PubMed PMID: 4656609; 1084 PubMed Central PMCID: PMCPMC1666567.

1085 21. Levi-Schaffer F, Tarrab-Hazdai R, Schryer MD, Arnon R, Smolarsky M. Isolation and 1086 partial characterization of the tegumental outer membrane of schistosomula of Schistosoma mansoni. Mol Biochem Parasitol. 1984;13(3):283-300. PubMed PMID: 6527692.

1088 22. Pearson MS, Becker L, Driguez P, Young ND, Gaze S, Mendes T, et al. Of monkeys and men: immunomic profiling of sera from humans and non-human primates resistant to schistosomiasis reveals novel potential vaccine candidates. Front Immunol. 2015;6:213. doi: 10.3389/fimmu.2015.00213. PubMed PMID: 25999951; PubMed Central PMCID: PMCPMC4419842.

1093 23. Jones AK, Bentley GN, Oliveros Parra WG, Agnew A. Molecular characterization of an 1094 acetylcholinesterase implicated in the regulation of glucose scavenging by the parasite Schistosoma. FASEB J. 2002;16(3):441-3. doi: 10.1096/fj.01-0683fje. PubMed PMID: 11821256.

1097 24. Crabtree JE, Wilson RA. Schistosoma mansoni: a scanning electron microscope study of 1098 the developing schistosomulum. Parasitol. 1980;81(Pt 3):553-64. PubMed PMID: 7232034.

1099 25. You H, Gobert GN, Du X, Pali G, Cai P, Jones MK, et al. Functional characterisation of 1100 Schistosoma japonicum acetylcholinesterase. Parasit Vectors. 2016;9(1):328. doi: 1101 10.1186/s13071-016-1615-1. PubMed PMID: 27283196; PubMed Central PMCID: 1102 PMCPMC4901427.

1103 26. You H, Liu C, Du X, Nawaratna S, Rivera V, Harvie M, et al. Suppression of 1104 Schistosoma japonicum Acetylcholinesterase Affects Parasite Growth and Development. Int J 1105 Mol Sci. 2018;19(8):2426-. doi: 10.3390/ijms19082426. PubMed PMID: 30115897; PubMed 1106 Central PMCID: PMCPMC6121427.

1107 27. Gang SS, Castelletto ML, Bryant AS, Yang E, Mancuso N, Lopez JB, et al. Targeted 1108 mutagenesis in a human-parasitic nematode. PLoS Pathog. 2017;13(10):e1006675. doi: 
1109 10.1371/journal.ppat.1006675. PubMed PMID: 29016680; PubMed Central PMCID: 1110 PMCPMC5650185.

1111 28. Clement K, Rees H, Canver MC, Gehrke JM, Farouni R, Hsu JY, et al. CRISPResso2 1112 provides accurate and rapid genome editing sequence analysis. Nat Biotechnol. 2019;37(3):2241113 6. doi: 10.1038/s41587-019-0032-3. PubMed PMID: 30809026; PubMed Central PMCID: 1114 PMCPMC6533916.

1115 29. Anthony BJ, Ramm GA, McManus DP. Role of resident liver cells in the pathogenesis of 1116 schistosomiasis. Trends Parasitol. 2012;28(12):572-9. doi: 10.1016/j.pt.2012.09.005. PubMed 1117 PMID: 23099112.

1118 30. Carson JP, Ramm GA, Robinson MW, McManus DP, Gobert GN. Schistosome-Induced 1119 Fibrotic Disease: The Role of Hepatic Stellate Cells. Trends Parasitol. 2018;34(6):524-40. doi: 1120 10.1016/j.pt.2018.02.005. PubMed PMID: 29526403.

1121 31. Xu L, Hui AY, Albanis E, Arthur MJ, O'Byrne SM, Blaner WS, et al. Human hepatic 1122 stellate cell lines, LX-1 and LX-2: new tools for analysis of hepatic fibrosis. Gut. 1123 2005;54(1):142-51. doi: 10.1136/gut.2004.042127. PubMed PMID: 15591520; PubMed Central 1124 PMCID: PMCPMC1774377.

1125 32. Boros DL, Warren KS. Delayed hypersensitivity-type granuloma formation and dermal 1126 reaction induced and elicited by a soluble factor isolated from Schistosoma mansoni eggs. J Exp 1127 Med. 1970;132(3):488-507. doi: 10.1084/jem.132.3.488. PubMed PMID: 5535626; PubMed 1128 Central PMCID: PMCPMC2138804.

1129 33. Pu Zhang FM. IgE: a Key Antibody in Schistosoma Infection Electronic Journal of 1130 Biology. 2006;2(1):11-4.

1131 34. Barron L, Wynn TA. Macrophage activation governs schistosomiasis-induced 1132 inflammation and fibrosis. Eur J Immunol. 2011;41(9):2509-14. doi: 10.1002/eji.201141869. 1133 PubMed PMID: 21952807; PubMed Central PMCID: PMCPMC3408543.

1134 35. Costain AH, MacDonald AS, Smits HH. Schistosome Egg Migration: Mechanisms, 1135 Pathogenesis and Host Immune Responses. Front Immunol. 2018;9:3042. doi: 1136 10.3389/fimmu.2018.03042. PubMed PMID: 30619372; PubMed Central PMCID:

\section{PMCPMC6306409.}

1138 36. Mayer JU, Demiri M, Agace WW, MacDonald AS, Svensson-Frej M, Milling SW. 1139 Different populations of CD11b(+) dendritic cells drive Th2 responses in the small intestine and 1140 colon. Nat Commun. 2017;8:15820. doi: 10.1038/ncomms15820. PubMed PMID: 28598427; 1141 PubMed Central PMCID: PMCPMC5472728.

1142 37. Mali P, Yang L, Esvelt KM, Aach J, Guell M, DiCarlo JE, et al. RNA-guided human 1143 genome engineering via Cas9. Science. 2013;339(6121):823-6. doi: 10.1126/science.1232033. 1144 PubMed PMID: 23287722; PubMed Central PMCID: PMCPMC3712628.

1145 38. Wang H, Yang H, Shivalila CS, Dawlaty MM, Cheng AW, Zhang F, et al. One-step 1146 generation of mice carrying mutations in multiple genes by CRISPR/Cas-mediated genome 1147 engineering. Cell. 2013;153(4):910-8. doi: 10.1016/j.cell.2013.04.025. PubMed PMID: 1148 23643243; PubMed Central PMCID: PMCPMC3969854. 
1149 39. Fox BA, Ristuccia JG, Gigley JP, Bzik DJ. Efficient gene replacements in Toxoplasma gondii strains deficient for nonhomologous end joining. Eukaryotic cell. 2009;8(4):520-9. 40. Huynh M-H, Carruthers VB. Tagging of endogenous genes in a Toxoplasma gondii strain lacking Ku80. Eukaryotic cell. 2009;8(4):530-9.

1153 41. Fox BA, Falla A, Rommereim LM, Tomita T, Gigley JP, Mercier C, et al. Type II 1154 Toxoplasma gondii KU80 knockout strains enable functional analysis of genes required for cyst 1155 development and latent infection. Eukaryotic cell. 2011;10(9):1193-206.

1156 42. Takata M, Sasaki MS, Sonoda E, Morrison C, Hashimoto M, Utsumi H, et al. 1157 Homologous recombination and non-homologous end-joining pathways of DNA double-strand 1158 break repair have overlapping roles in the maintenance of chromosomal integrity in vertebrate 1159 cells. EMBO J. 1998;17(18):5497-508. doi: 10.1093/emboj/17.18.5497. PubMed PMID: 1160 9736627; PubMed Central PMCID: PMCPMC1170875.

1161 43. Gardner MJ, Hall N, Fung E, White O, Berriman M, Hyman RW, et al. Genome 1162 sequence of the human malaria parasite Plasmodium falciparum. Nature. 2002;419(6906):498.

1163 44. Aravind L, Iyer LM, Wellems TE, Miller LH. Plasmodium biology: genomic gleanings. 1164 Cell. 2003;115(7):771-85.

1165 45. Kirkman LA, Lawrence EA, Deitsch KW. Malaria parasites utilize both homologous 1166 recombination and alternative end joining pathways to maintain genome integrity. Nucleic acids 1167 research. 2013;42(1):370-9.

1168 46. Lee AH, Symington LS, Fidock DA. DNA repair mechanisms and their biological roles 1169 in the malaria parasite Plasmodium falciparum. Microbiol Mol Biol Rev. 2014;78(3):469-86.

1170 47. Janssen BD, Chen Y-P, Molgora BM, Wang SE, Simoes-Barbosa A, Johnson PJ. 1171 CRISPR/Cas9-mediated gene modification and gene knock out in the human-infective parasite 1172 Trichomonas vaginalis. Scientific reports. 2018;8(1):270.

1173 48. Vinayak S, Pawlowic MC, Sateriale A, Brooks CF, Studstill CJ, Bar-Peled Y, et al. 1174 Genetic modification of the diarrhoeal pathogen Cryptosporidium parvum. Nature. 1175 2015;523(7561):477.

1176 49. Donald RG, Roos DS. Homologous recombination and gene replacement at the 1177 dihydrofolate reductase-thymidylate synthase locus in Toxoplasma gondii. Molecular and 1178 biochemical parasitology. 1994;63(2):243-53.

1179 50. Craver MPJ, Knoll LJ. Increased efficiency of homologous recombination in Toxoplasma 1180 gondii dense granule protein 3 demonstrates that GRA3 is not necessary in cell culture but does 1181 contribute to virulence. Molecular and biochemical parasitology. 2007;153(2):149-57.

1182 51. Xu X, Gao D, Wang P, Chen J, Ruan J, Xu J, et al. Efficient homology-directed gene 1183 editing by CRISPR/Cas9 in human stem and primary cells using tube electroporation. Sci Rep. 1184 2018;8(1):11649. doi: 10.1038/s41598-018-30227-w. PubMed PMID: 30076383; PubMed 1185 Central PMCID: PMCPMC6076306.

1186 52. McVeigh P, Maule AG. Can CRISPR help in the fight against parasitic worms? Elife. 1187 2019;8. doi: 10.7554/eLife.44382. PubMed PMID: 30702425; PubMed Central PMCID: 1188 PMCPMC6355191. 
1189 53. Gang SS, Castelletto ML, Bryant AS, Yang E, Mancuso N, Lopez JB, et al. Targeted mutagenesis in a human-parasitic nematode. PLoS pathogens. 2017;13(10):e1006675.

1191

54. Chiu H, Schwartz HT, Antoshechkin I, Sternberg PW. Transgene-free genome editing in Caenorhabditis elegans using CRISPR-Cas. Genetics. 2013;195(3):1167-71. doi: 10.1534/genetics.113.155879. PubMed PMID: 23979577; PubMed Central PMCID: PMCPMC3813845.

55. Neill PJ, Smith JH, Doughty BL, Kemp M. The ultrastructure of the Schistosoma mansoni egg. The American journal of tropical medicine and hygiene. 1988;39(1):52-65.

56. Ashton PD, Harrop R, Shah B, Wilson RA. The schistosome egg: development and secretions. Parasitology. 2001;122(Pt 3):329-38. doi: 10.1017/s0031182001007351. PubMed PMID: 11289069. transgenesis and insertional mutagenesis in Schistosoma mansoni mediated by murine leukemia virus. PLoS pathogens. 2012;8(7):e1002820. The embryonic development of Schistosoma mansoni eggs: proposal for a new staging system. 19415326.

59. Chuah C, Jones MK, Burke ML, McManus DP, Gobert GN. Cellular and chemokinemediated regulation in schistosome-induced hepatic pathology. Trends Parasitol. 2014;30(3):141-50. doi: 10.1016/j.pt.2013.12.009. PubMed PMID: 24433721. of the Immune Response by Nematode Secreted Acetylcholinesterase Revealed by Heterologous

1212 Expression in Trypanosoma musculi. PLoS Pathog. 2016;12(11):e1005998. doi: 10.1371/journal.ppat.1005998. PubMed PMID: 27802350; PubMed Central PMCID:

1214 PMCPMC5089771.

1215 61. Hsieh CS, Heimberger AB, Gold JS, O'Garra A, Murphy KM. Differential regulation of $1216 \mathrm{~T}$ helper phenotype development by interleukins 4 and 10 in an alpha beta T-cell-receptor 1217 transgenic system. Proc Natl Acad Sci U S A. 1992;89(13):6065-9. PubMed PMID: 1385868; 1218 PubMed Central PMCID: PMCPMC49438.

1219 62. Rincon M, Anguita J, Nakamura T, Fikrig E, Flavell RA. Interleukin (IL)-6 directs the 1220 differentiation of IL-4-producing CD4+ T cells. J Exp Med. 1997;185(3):461-9. PubMed PMID: 9053446; PubMed Central PMCID: PMCPMC2196041.

1222 63. Trinchieri G. Cytokines acting on or secreted by macrophages during intracellular 1223 infection (IL-10, IL-12, IFN-gamma). Curr Opin Immunol. 1997;9(1):17-23. PubMed PMID: 12249039773.

1225 64. Abbas AK, Murphy KM, Sher A. Functional diversity of helper T lymphocytes. Nature. 1226 1996;383(6603):787-93. doi: 10.1038/383787a0. PubMed PMID: 8893001.

1227 65. Koslowski N, Sombetzki M, Loebermann M, Engelmann R, Grabow N, Osterreicher CH, 1228 et al. Single-sex infection with female Schistosoma mansoni cercariae mitigates hepatic fibrosis 
1229 after secondary infection. PLoS Negl Trop Dis. 2017;11(5):e0005595. doi: 1230 10.1371/journal.pntd.0005595. PubMed PMID: 28542175; PubMed Central PMCID: 1231 PMCPMC5453606.

1232 66. Nair MG, Du Y, Perrigoue JG, Zaph C, Taylor JJ, Goldschmidt M, et al. Alternatively 1233 activated macrophage-derived RELM- $\{$ alpha $\}$ is a negative regulator of type 2 inflammation in 1234 the lung. J Exp Med. 2009;206(4):937-52. doi: 10.1084/jem.20082048. PubMed PMID: 1235 19349464; PubMed Central PMCID: PMCPMC2715126.

1236 67. McManus DP, Dunne DW, Sacko M, Utzinger J, Vennervald BJ, Zhou XN. 1237 Schistosomiasis. Nat Rev Dis Primers. 2018;4(1):13. doi: 10.1038/s41572-018-0013-8. PubMed 1238 PMID: 30093684.

1239 68. Chuah C, Jones MK, Burke ML, Owen HC, Anthony BJ, McManus DP, et al. Spatial and 1240 temporal transcriptomics of Schistosoma japonicum-induced hepatic granuloma formation 1241 reveals novel roles for neutrophils. J Leukoc Biol. 2013;94(2):353-65. doi: 10.1189/jlb.1212653. 1242 PubMed PMID: 23709687.

1243 69. Ariyaratne A, Finney CAM. Eosinophils and Macrophages within the Th2-Induced 1244 Granuloma: Balancing Killing and Healing in a Tight Space. Infect Immun. 2019;87(10). doi: 1245 10.1128/IAI.00127-19. PubMed PMID: 31285249; PubMed Central PMCID: 1246 PMCPMC6759305.

1247 70. Almadi MA, Aljebreen AM, Sanai FM, Marcus V, Almeghaiseeb ES, Ghosh S. New 1248 insights into gastrointestinal and hepatic granulomatous disorders. Nat Rev Gastroenterol 1249 Hepatol. 2011;8(8):455-66. doi: 10.1038/nrgastro.2011.115. PubMed PMID: 21818145.

1250 71. Chatterjee S, Mbaye A, Alfidja AT, Weyler J, Scott JT, Van Damme P, et al. Circulating 1251 levels of the neuropeptide hormone somatostatin may determine hepatic fibrosis in Schistosoma 1252 mansoni infections. Acta Trop. 2004;90(2):191-203. doi: 10.1016/j.actatropica.2003.12.002. 1253 PubMed PMID: 15177146.

1254 72. Anthony BJ, James KR, Gobert GN, Ramm GA, McManus DP. Schistosoma japonicum 1255 Eggs Induce a Proinflammatory, Anti-Fibrogenic Phenotype in Hepatic Stellate Cells. PLoS One. 1256 2013;8(6):e68479. doi: 10.1371/journal.pone.0068479. PubMed PMID: 23840855; PubMed 1257 Central PMCID: PMCPMC3686876.

1258 73. Lan Z, Chen L, Fu Q, Ji W, Wang S, Liang Z, et al. Paeoniflorin attenuates amyloid-beta 1259 peptide-induced neurotoxicity by ameliorating oxidative stress and regulating the NGF-mediated 1260 signaling in rats. Brain Res. 2013;1498:9-19. doi: 10.1016/j.brainres.2012.12.040. PubMed 1261 PMID: 23295189.

1262 74. Chu D, Luo Q, Li C, Gao Y, Yu L, Wei W, et al. Paeoniflorin inhibits TGF-beta11263 mediated collagen production by Schistosoma japonicum soluble egg antigen in vitro. 1264 Parasitology. 2007;134(Pt 11):1611-21. doi: 10.1017/S0031182007002946. PubMed PMID: 126517524166.

1266 75. Dalton JP, Day SR, Drew AC, Brindley PJ. A method for the isolation of schistosome 1267 eggs and miracidia free of contaminating host tissues. Parasitology. 1997;115 ( Pt 1):29-32. 1268 PubMed PMID: 9226954. 
1269 76. You H, Zhang W, Jones MK, Gobert GN, Mulvenna J, Rees G, et al. Cloning and 1270 characterisation of Schistosoma japonicum insulin receptors. PLoS One. 2010;5(3):e9868. doi: 10.1371/journal.pone.0009868. PubMed PMID: 20352052; PubMed Central PMCID:

1272 PMCPMC2844434.

1273 77. Mann VH, Morales ME, Rinaldi G, Brindley PJ. Culture for genetic manipulation of 1274 developmental stages of Schistosoma mansoni. Parasitology. 2010;137(3):451-62. doi: 1275 10.1017/S0031182009991211. PubMed PMID: 19765348; PubMed Central PMCID: 1276 PMCPMC3042131.

1277 78. Oliveros JC, Franch M, Tabas-Madrid D, San-Leon D, Montoliu L, Cubas P, et al. 1278 Breaking-Cas-interactive design of guide RNAs for CRISPR-Cas experiments for ENSEMBL 1279 genomes. Nucleic Acids Res. 2016;44(W1):W267-71. doi: 10.1093/nar/gkw407. PubMed PMID: 1280 27166368; PubMed Central PMCID: PMCPMC4987939.

1281 79. You H, McManus DP, Hu W, Smout MJ, Brindley PJ, Gobert GN. Transcriptional 1282 responses of in vivo praziquantel exposure in schistosomes identifies a functional role for 1283 calcium signalling pathway member CamKII. PLoS Pathog. 2013;9(3):e1003254. doi: 1284 10.1371/journal.ppat.1003254. PubMed PMID: 23555262; PubMed Central PMCID: 1285 PMCPMC3610926.

1286 80. Liang CC, Park AY, Guan JL. In vitro scratch assay: a convenient and inexpensive 1287 method for analysis of cell migration in vitro. Nat Protoc. 2007;2(2):329-33. doi: 1288 10.1038/nprot.2007.30. PubMed PMID: 17406593.

1289 81. Ranasinghe SL, Boyle GM, Fischer K, Potriquet J, Mulvenna JP, McManus DP. Kunitz 1290 type protease inhibitor EgKI-1 from the canine tapeworm Echinococcus granulosus as a 1291 promising therapeutic against breast cancer. PLoS One. 2018;13(8):e0200433. doi: 1292 10.1371/journal.pone.0200433. PubMed PMID: 30169534; PubMed Central PMCID: 1293 PMCPMC6118354.

1294 82. Edungbola LD, Schiller EL. Histopathology of hepatic and pulmonary granulomata 1295 experimentally induced with eggs of Schistosoma mansoni. J Parasitol. 1979;65(2):253-61. 1296 PubMed PMID: 571911.

1297 83. Ricciardi A, Dalton JP, Ndao M. Evaluation of the immune response and protective 1298 efficacy of Schistosoma mansoni Cathepsin B in mice using CpG dinucleotides as adjuvant. 1299 Vaccine. 2015;33(2):346-53. doi: 10.1016/j.vaccine.2014.11.016. PubMed PMID: 25448114.

1300 84. Hagen J, Young ND, Every AL, Pagel CN, Schnoeller C, Scheerlinck JP, et al. Omega-1 1301 knockdown in Schistosoma mansoni eggs by lentivirus transduction reduces granuloma size in 1302 vivo. Nat Commun. 2014;5:5375. doi: 10.1038/ncomms6375. PubMed PMID: 25400038; 1303 PubMed Central PMCID: PMCPMC4243216.

1304 85. Hoffmann KF, Johnston DA, Dunne DW. Identification of Schistosoma mansoni gender1305 associated gene transcripts by cDNA microarray profiling. Genome Biol. 1306 2002;3(8):RESEARCH0041. PubMed PMID: 12186648.

1307 86. You H, Zhang W, Moertel L, McManus DP, Gobert GN. Transcriptional profiles of adult 1308 male and female Schistosoma japonicum in response to insulin reveal increased expression of 
1309 genes involved in growth and development. Int J Parasitol. 2009;39(14):1551-9. doi: 1310 10.1016/j.ijpara.2009.06.006. PubMed PMID: 19596015.

1311 87. Burke ML, McManus DP, Ramm GA, Duke M, Li Y, Jones MK, et al. Temporal 1312 expression of chemokines dictates the hepatic inflammatory infiltrate in a murine model of 1313 schistosomiasis. PLoS Negl Trop Dis. 2010;4(2):e598. doi: 10.1371/journal.pntd.0000598. 1314 PubMed PMID: 20161726; PubMed Central PMCID: PMCPMC2817718.

1315 88. Perry CR, Burke ML, Stenzel DJ, McManus DP, Ramm GA, Gobert GN. Differential 1316 expression of chemokine and matrix re-modelling genes is associated with contrasting 1317 schistosome-induced hepatopathology in murine models. PLoS Negl Trop Dis. 2011;5(6):e1178.

1318 Epub 2011/06/15. doi: 10.1371/journal.pntd.0001178

1319 PNTD-D-10-00215 [pii]. PubMed PMID: 21666794; PubMed Central PMCID: PMC3110159.

1320 89. Chuah C, Jones MK, McManus DP, Nawaratna SK, Burke ML, Owen HC, et al. 1321 Characterising granuloma regression and liver recovery in a murine model of schistosomiasis 1322 japonica. Int J Parasitol. 2016;46(4):239-52. doi: 10.1016/j.ijpara.2015.12.004. PubMed PMID: 132326812024. 


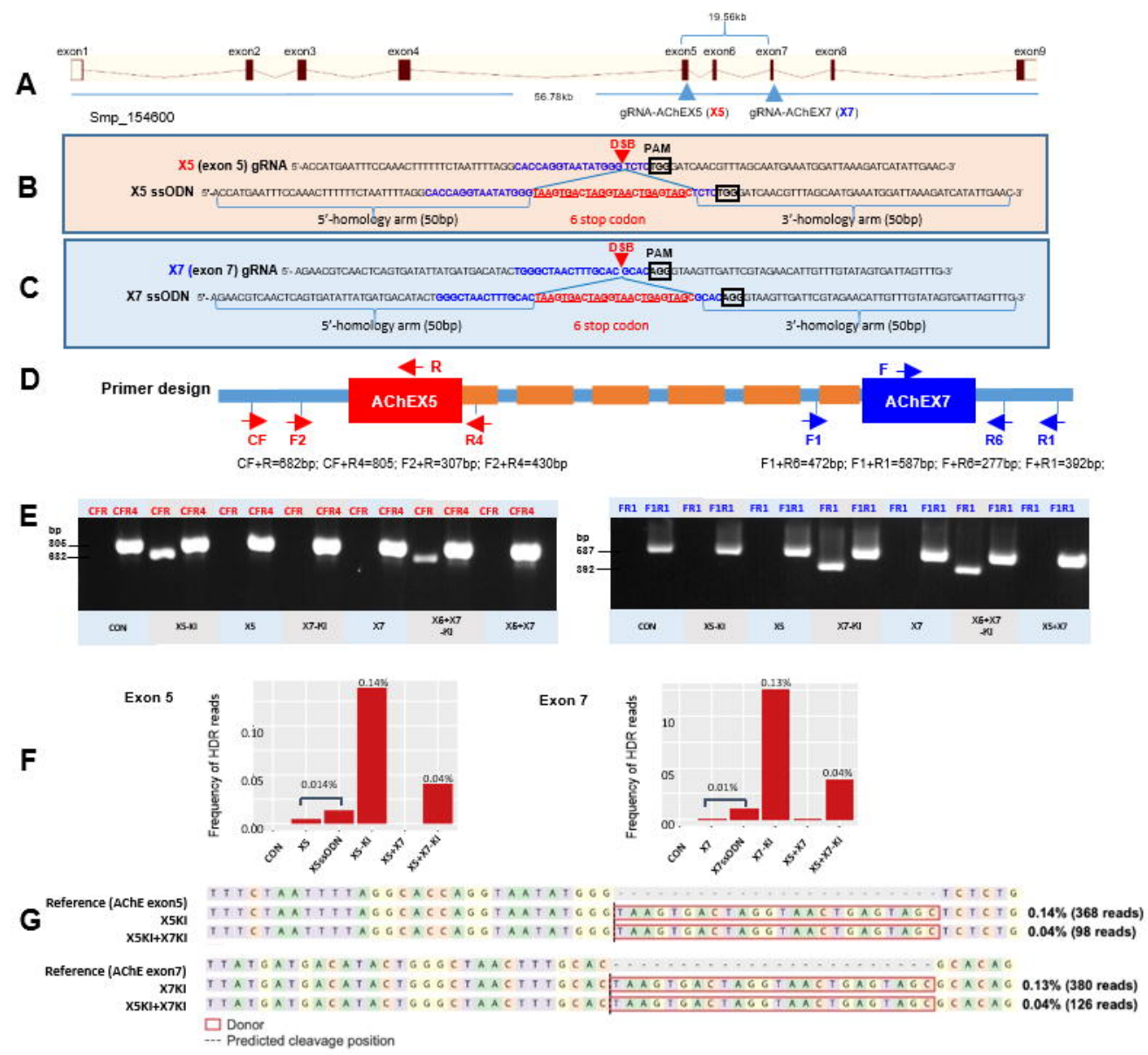



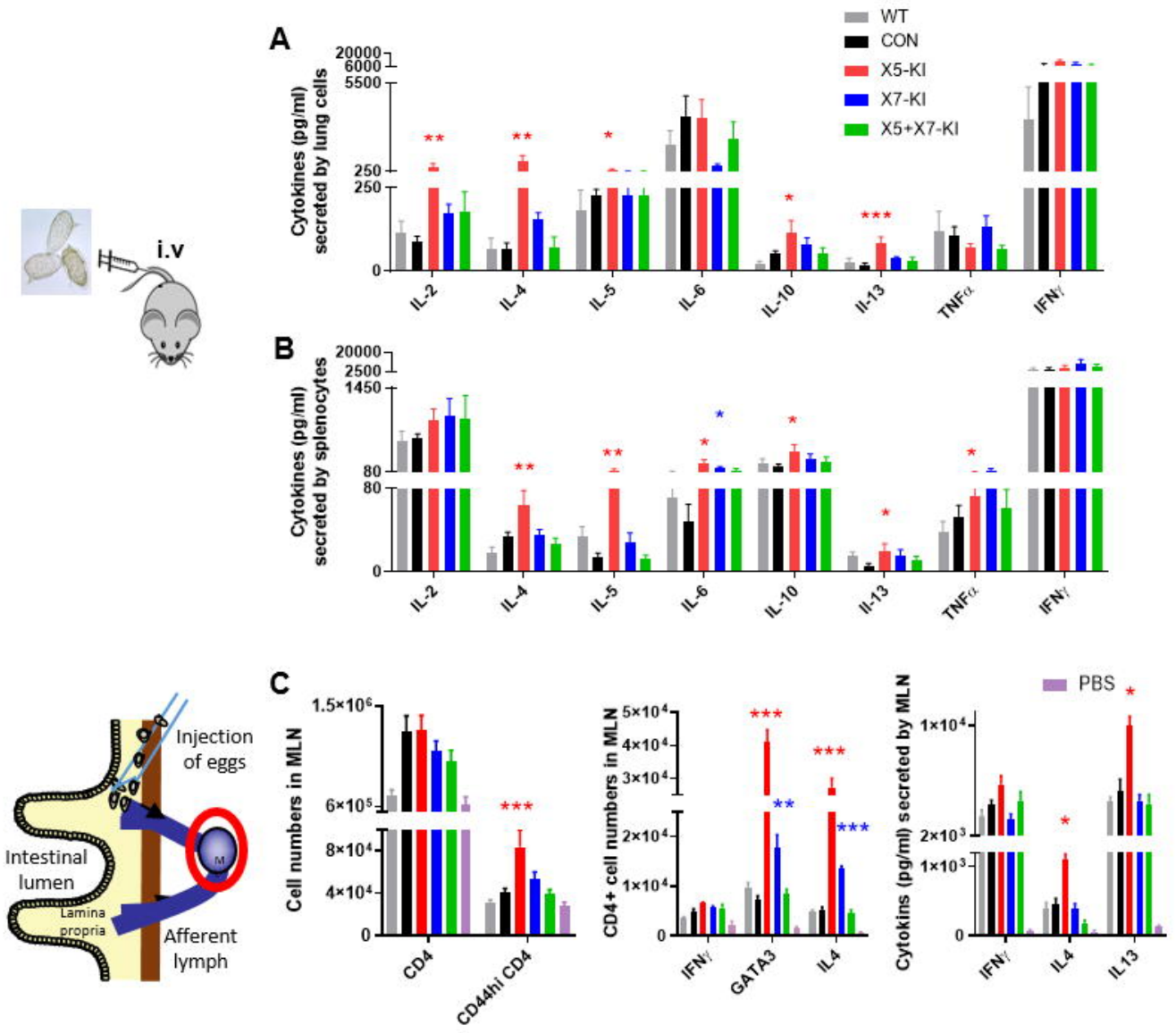